\title{
Synthesis, In Vitro Antiproliferative Evaluation and Molecular Docking of New tetrazole-chalcone and tetrazole-pyrazoline Hybrids
}

\author{
Heidi S. Abd ElMonaem¹, Naglaa I. Abdel-Aziz ${ }^{1,2^{*}}$, Mohammad A. Morsy ${ }^{2,3}$, Farid A. Badria ${ }^{4}$, Fardous ElSenduny ${ }^{5}$, \\ Mahmoud B. El-Ashmawy ${ }^{1}$, Mohamed A. Moustafa ${ }^{1,6}$ \\ ${ }^{1}$ Department of Medicinal Chemistry, Faculty of Pharmacy, Mansoura University, Mansoura 35516, Egypt. \\ ${ }^{2}$ Department of Pharmaceutical Chemistry, Faculty of Pharmacy, Delta University for Science and Technology, Egypt. \\ ${ }^{3}$ Department of Pharmaceutical Organic Chemistry, Faculty of Pharmacy, Mansoura University, Mansoura, 35516, Egypt. \\ ${ }^{4}$ Department of Pharmacognosy, Faculty of Pharmacy, Mansoura University, Mansoura 35516, Egypt. \\ ${ }^{5}$ Department of Biochemistry, Faculty of Science, Mansoura University, Mansoura 35516, Egypt. \\ ${ }^{6}$ Department of Pharmaceutical Chemistry, Faculty of Pharmacy, King Abdulaziz University, Jeddah 21589, Kingdom of Saudi Arabia.
}

\section{ARTICLE INFO \\ Article history: \\ Received on: 24/02/2018 \\ Accepted on: 26/03/2018 \\ Available online: $30 / 05 / 2018$}

\section{Key words:}

Tetrazole, Chalcone,

Pyrazoline, Antiproliferative,

Molecular docking.

\begin{tabular}{l}
\hline ABSTRACT \\
New hybrids of tetrazole moiety with different chalcone derivatives were synthesized. The reaction of these \\
chalcones with hydrazine hydrate resulted in the formation of tetrazole-pyrazoline hybrids. Evaluation of the in vitro \\
antiproliferative activity of all newly synthesized hybrids against three cancer cell lines and Vero- $B$ normal cell line, \\
using MTT-based assay, was performed. Most of the chalcone derivatives exerted superior activity against colon $H C T$ - \\
116 and prostate $P C-3$ cell lines, in comparison with cisplatin $\left(\mathrm{IC}_{50}=20\right.$ and $\left.5 \mu \mathrm{g} / \mathrm{ml}\right)$ and 5 -FU $\left(\mathrm{IC}_{50}=17.3\right.$ and 21.4 \\
$\mu \mathrm{g} / \mathrm{ml})$, respectively. Compound $\mathbf{5 a}$ was found to be the most active antiproliferative agent against colon $H C T-116$ \\
and prostate $P C-3$ cell lines $\left(\mathrm{IC}_{50}=0.6\right.$ and $\left.1.6 \mu \mathrm{g} / \mathrm{ml}\right)$ with high selectivity indices $(\mathrm{SI}=6.66$ and 2.50$)$, respectively. \\
Compound $\mathbf{8 f}$, in particular, displayed a wider spectrum of activity that included, in addition, excellent effect against \\
breast $M C F-7$ cell lines with $\mathrm{SI}=2.75$. The available docking results revealed a good binding of $\mathbf{5 b}$ with HDAC2 and \\
CYP17A1 that endorses the in vitro biological activity against the tested colon and prostate cell lines.
\end{tabular}

\section{INTRODUCTION}

Cancer designates a group of diseases characterized by malignant cells that grow and divide uncontrollably. The abnormal cells have the capacity to spread and invade surrounding tissues via a metastasis process (Vogelstein et al., 2013); and the abnormal mutations to DNA within cells may be either inherited or caused by carcinogenic agents (Fischer et al., 2005). The traditional cancer treatment includes surgery, chemotherapy and radiation therapy; and the ideal goal of chemotherapy is to specifically target cancer cells without affecting normal cells, to avoid toxic side effects. Searching for new compounds with potent and selective anticancer activities is highly demanded. Literature survey highlighted the

*Corresponding Author

Naglaa I. Abdel-Aziz, Department of Medicinal Chemistry, Faculty of Pharmacy, Mansoura University, Mansoura 35516, Egypt; Department of Pharmaceutical Chemistry, Faculty of Pharmacy, Delta University for Science and Technology.E-mail: naglaabdalaziz2005@yahoo.com importance of tetrazole-containing compounds (A; Figure 1) as antitumor agents (Bayomi et al., 2016; Arshad et al., 2014; Jedhe et al., 2013; Bhaskar and Mohite, 2010). On the other hand, compounds with Chalcone-based structure (B; Figure 1) were described as cytotoxic agents that act via different mechanisms including induction of apoptosis and inhibition of cell proliferation (Sankappa Rai et al., 2015; Bayomi et al., 2015; Bayomi et al., 2013; de Vasconcelos et al., 2013; Sharma et al., 2013; Murad et al., 2012). Some pyrazoline derivatives (Yuan et al., 2014; Viale et al., 2013; Nitulescu et al., 2013) were also reported (C; Figure 1) to inhibit different enzymes that are involved in the cell division.

In view of the previous findings, our research group got interested to design new hybrid molecules, where the tetrazole nucleus and chalcone or pyrazoline functionality were incorporated into the single compound to be evaluated for antiproliferative activity (Figure 1). We thought that co-occurrence of tetrazole and chalcone/pyrazoline pharmacophores should result in new derivatives with the promising biological profile. 
The antiproliferative activity of the newly synthesized compounds was carried out using MTT-based assay against human colon cancer (HCT-116), prostate cancer $(P C-3)$ and human breast cancer $(M C F-7)$ cell lines along with the normal kidney of African green monkey (Vero B) cell line. A molecular docking simulation was performed to explore the binding interactions with different enzymes involved in cell proliferation, namely: histone deacetylase 2 enzyme (HDAC2), cytochrome P450 17A1 and 5,10-methenyltetrahydrofolate synthetase (MTHFS).

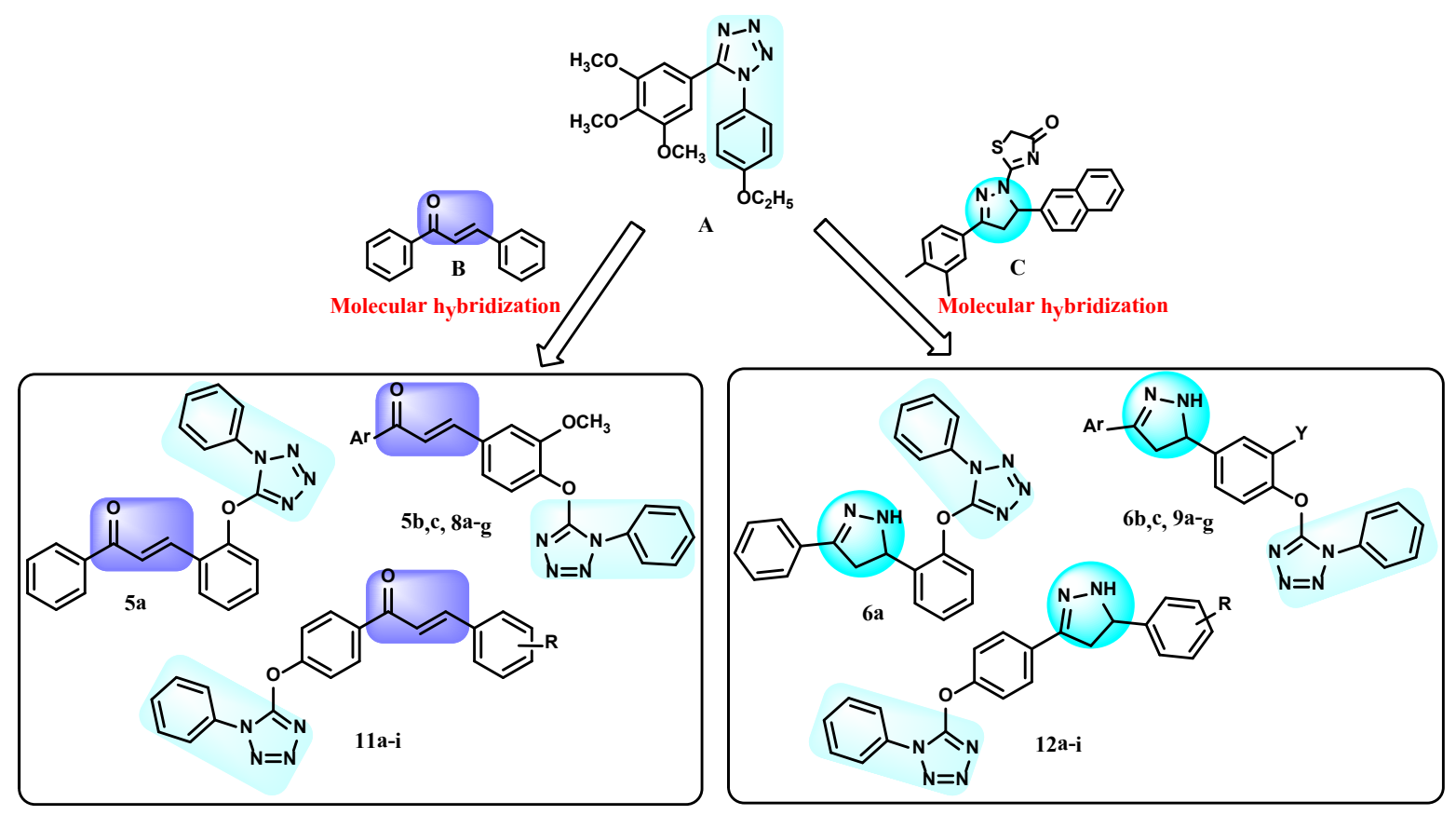

Fig. 1: Reported antitumor compounds (A-C); and the designed tetrazole-chalcone and tetrazole-pyrazoline hybrids.

\section{EXPERIMENTAL PROTOCOLS}

\section{Chemistry}

Melting points $\left({ }^{\circ} \mathrm{C}\right.$, uncorrected) were measured using Fisher-Johns apparatus. Elemental analyses (C, H, N) were carried out at the microanalytical unit, Cairo University. IR spectra $(\mathrm{KBr})$ were attained using Mattson 5000 FT-IR spectrometer $(v$ in $\mathrm{cm}^{-1}$ ) at Faculty of Pharmacy, Mansoura University and the results were expressed in wave number $\left(\mathrm{cm}^{-1}\right) .{ }^{1} \mathrm{H}$ NMR and ${ }^{13} \mathrm{C}$ NMR spectra were obtained on FT-NMR spectrometer (300 MHz) Gemini Varian, using TMS as internal standard and DMSO as a solvent (chemical shifts in $\delta$ units, ppm), at Cairo University. MS measurements were performed on JEOL JMS-600H spectrometer, Cairo University. The purities of the compounds were checked by thin layer chromatography (TLC) on silica gel G (Merck) and spots were visualized by irradiation with ultraviolet light (UV; $254 \mathrm{~nm}$ ). All chemicals, reagents, and solvents were purchased from Aldrich Chemical Company and used as received. The known compounds, E-3-(2-Hydroxyphenyl)-1-phenylprop-2-en-1-one (3a) (Pan et al., 2013), E-1-(3,4-Dimethoxyphenyl)-3-(4-hydroxyphenyl)prop2-en-1-one (3b) (Cai et al., 2017), E-1-(3,4-Dimethoxyphenyl)3-(4-hydroxy-3-methoxyphenyl) prop-2-en-1-one (3c) (Pathak et al., 2014), 3-Methoxy-4-((1-phenyl-1H-tetrazol-5-yl)oxy) benzaldehyde (7) (Buonerba et al., 2017), 4-((1-phenyl-1Htetrazol-5-yl)oxy)acetophenone (10) (Eisa et al., 1990), were prepared according to the reported procedures.
General procedure for synthesis of (E)-1-phenyl-3-(2-((1-phenyl1H-tetrazol-5-yl)oxy)phenyl)prop-2-en-1-one (5a) and (E)-1(3,4-dimethoxyphenyl)-3-(4-((1-phenyl-1H-tetrazol-5-yl)oxy)-3(un)substituted phenyl)prop-2-en-1-ones $(\mathbf{5} \boldsymbol{b}, \boldsymbol{c})$ (Scheme 1)

5-Chloro-1-phenyltetrazole (4) (1.8 g, $10 \mathrm{mmol})$ was added, with stirring, to a mixture of the chalcone derivative (3a-c) $(10 \mathrm{mmol})$ and anhydrous $\mathrm{K}_{2} \mathrm{CO}_{3}(2.0 \mathrm{~g}, 15 \mathrm{mmol})$ in DMF $(15 \mathrm{~mL})$. The reaction mixture was stirred for 24 hours at room temperature and then diluted with water $(30 \mathrm{~mL})$. The precipitated solid product was collected by filtration, washed with water, dried and crystallized from ethanol to give compounds 5a-c.

\section{E-1-phenyl-3-(2-((1-phenyl-1H-tetrazol-5-yl)oxy)phenyl)prop-2- en-1-one (5a)}

Yield 68\%; mp 151-152 ${ }^{\circ} \mathrm{C}$; IR; $1665(\mathrm{C}=\mathrm{O}), 1604$ $(\mathrm{C}=\mathrm{C}), 1182(\mathrm{C}-\mathrm{O}) .{ }^{1} \mathrm{H}$ NMR; 6.81-7.59 (m, 11H, ArH), $7.71(\mathrm{~d}$, $1 \mathrm{H}, \mathrm{CO}-\mathrm{C} \underline{\mathrm{H}}=\mathrm{CH}, J=14), 7.77-7.98(\mathrm{~m}, 3 \mathrm{H}, \mathrm{ArH}), 8.12(\mathrm{~d}, 1 \mathrm{H}$, $\mathrm{CO}-\mathrm{CH}=\mathrm{C} \underline{\mathrm{H}}, J=14)$. Anal. Calcd for $\mathrm{C}_{22} \mathrm{H}_{16} \mathrm{~N}_{4} \mathrm{O}_{2}(\%)$ : C, 71.73; H, 4.38; N, 15.21. Found: C, 72.03; H, 4.62; N, 15.38.

\section{E-1-(3,4-Dimethoxyphenyl)-3-(4-((1-phenyl-1H-tetrazol-5-yl) oxy)phenyl)prop-2-en-1-one (5b)}

Yield $61 \%$; mp $118-120^{\circ} \mathrm{C}$; IR; $1657(\mathrm{C}=\mathrm{O}), 1593$ $(\mathrm{C}=\mathrm{C}), 1161$ (C-O). ${ }^{1} \mathrm{H} \mathrm{NMR} ; 3.80\left(\mathrm{~s}, 6 \mathrm{H}, \mathrm{OCH}_{3}\right), 6.87-7.50$ $(\mathrm{m}, 9 \mathrm{H}, \mathrm{ArH}), 7.60(\mathrm{~d}, 1 \mathrm{H}, \mathrm{CO}-\mathrm{CH}=\mathrm{CH}, J=15), 7.66-7.69(\mathrm{~m}$, $3 \mathrm{H}, \mathrm{ArH}), 7.78$ (d, $1 \mathrm{H}, \mathrm{CO}-\mathrm{CH}=\mathrm{CH}, J=15),{ }^{13} \mathrm{C} \mathrm{NMR} ; 188.32$ $(\mathrm{C}=\mathrm{O}), 159.27,153.48,150.27,149.37,145.53,143.69,132.57$, 
$131.13,130.92,129.91,129.71,129.38,124.48,123.17,122.80$ (2C), 121.94, 122.59, 121.21, 112.59, 110.85, $56.16\left(\mathrm{OC}_{3}\right)$,
$56.13\left(\mathrm{O}_{-} \mathrm{H}_{3}\right)$. Anal. Calcd. for $\mathrm{C}_{24} \mathrm{H}_{20} \mathrm{~N}_{4} \mathrm{O}_{4}(\%): \mathrm{C}, 67.28 ; \mathrm{H}, 4.71$; N, 13.08. Found: C, 66.98; H, 4.76; N, 13.32 .

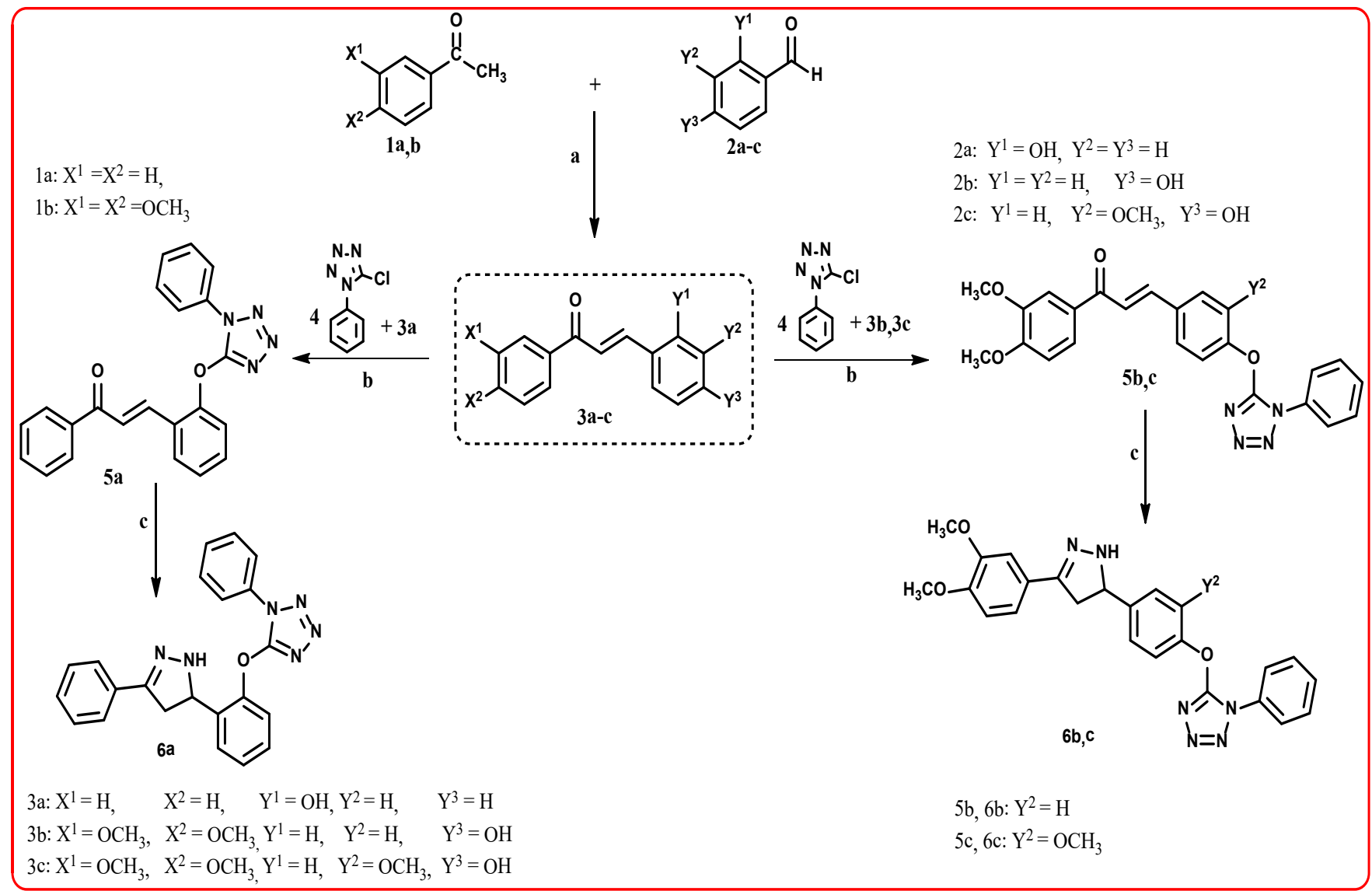

Scheme 1: Reagents and conditions: (a) $2.5 \% \mathrm{NaOH}, \mathrm{C}_{2} \mathrm{H}_{5} \mathrm{OH}$, RT.; (b) $\mathrm{K}_{2} \mathrm{CO}_{3}$, DMF, 24 h, RT. (c) $\mathrm{NH}_{2} \mathrm{NH}_{2} \cdot \mathrm{H}_{2} \mathrm{O}, \mathrm{C}_{2} \mathrm{H}_{5} \mathrm{OH}$, reflux, $10-15$ h.

E-1-(3,4-Dimethoxyphenyl)-3-(3-methoxy-4-((1-phenyl-1Htetrazol-5-yl)oxy)phenyl)prop-2-en-1-one (5c)

Yield 61\%; mp $132-133^{\circ} \mathrm{C}$; IR; $1658(\mathrm{C}=\mathrm{O}), 1597$ $(\mathrm{C}=\mathrm{C}), 1155(\mathrm{C}-\mathrm{O}) .{ }^{1} \mathrm{H}$ NMR; $3.80\left(\mathrm{~s}, 3 \mathrm{H}, \mathrm{OCH}_{3}\right), 3.87(\mathrm{~s}, 6 \mathrm{H}$, $\left.\mathrm{OCH}_{3}\right), 7.17-7.78(\mathrm{~m}, 8 \mathrm{H}, \mathrm{ArH}), 7.87(\mathrm{~d}, 1 \mathrm{H}, \mathrm{CO}-\mathrm{CH}=\mathrm{CH}, J=$ 14.5), 7.93-8.00 (m, 3H, ArH), 8.03 (d, 1H, CO-CH=CH, $J=$ 14.5). ${ }^{13} \mathrm{C}$ NMR; 191.79 (C=O), 159.27, 154.47, 153.31, 150.27, $148,82,146.09,143.23,141.62,130.49,130.42,129.94,129.82$, $128.63,123.46$ (2C), 123.57, 122.92, 122.56, 121.94, 113.41 $\left.110.88,56.42\left(\mathrm{O}_{\mathrm{CH}}\right)_{3}\right), 55.77\left(\mathrm{O}_{\underline{C H}}\right), 55.60\left(\mathrm{O}_{3} H_{3}\right)$. Anal. Calcd for $\mathrm{C}_{25} \mathrm{H}_{22} \mathrm{~N}_{4} \mathrm{O}_{5}(\%)$ : C, 65.49; $\mathrm{H}, 4.84 ; \mathrm{N}, 12.22$. Found: $\mathrm{C}, 65.68$; $\mathrm{H}, 4.52 ; \mathrm{N}, 11.95$.

General Procedure for Synthesis of 1-phenyl-5-(2-(3-phenyl4,5-dihydro-1H-pyrazol-5-yl)phenoxy)-1H-tetrazole (6a) and 5-(4-(3-(3,4-dimethoxyphenyl)-4,5-dihydro-1H-pyrazol-5-yl)-2(un)substituted-phenoxy)-1-phenyl-1H-tetrazoles (6b) and (6c) (Scheme 1)

A mixture of the chalcone derivative 5a-c $(10 \mathrm{mmol})$ and hydrazine monohydrate $(95 \%)(1.2 \mathrm{~mL}, 20 \mathrm{mmol})$ in ethanol $(30 \mathrm{~mL})$ was heated at reflux temperature for 10-15 hours. The reaction mixture was cooled, poured onto the crushed ice, and the obtained solid was collected by filtration, washed with water, dried and crystallized from ethanol to afford products $\mathbf{6 a - c .}$
1-Phenyl-5-(2-(3-phenyl-4,5-dihydro-1H-pyrazol-5-yl)phenoxy)1 H-tetrazole (6a)

Yield 60\%; mp 170-172 ${ }^{\circ} \mathrm{C}$; IR; $3336(\mathrm{NH}), 1590(\mathrm{C}=\mathrm{N})$, $1178(\mathrm{C}-\mathrm{O}) .{ }^{1} \mathrm{H}$ NMR; $3.12\left(\mathrm{dd}, 1 \mathrm{H}, \mathrm{C}_{4}\right.$ pyrazole, $J=11.8, J=$ 7.4), $3.88\left(\mathrm{dd}, 1 \mathrm{H}, \mathrm{C}_{4}\right.$ pyrazole, $\left.J=11.8, J=10.9\right), 4.86(\mathrm{dd}, 1 \mathrm{H}$, $\mathrm{C}_{5}$ pyrazole, $\left.J=10.9, J=7.4\right), 6.77-7.78(\mathrm{~m}, 14 \mathrm{H}, \mathrm{ArH}), 9.21(\mathrm{~s}$, $1 \mathrm{H}, \mathrm{N}-\mathrm{H}) . \mathrm{MS} \mathrm{m} / \mathrm{z}(\%) ; 382.41\left(\mathrm{M}^{+}, 2.11\right), 380.41$ (1.95), 378.94 (1.12), 282.09 (27.24), 265.26 (100), 256.20 (84.51), 236.98 (11.68). Anal. Calcd for $\mathrm{C}_{22} \mathrm{H}_{18} \mathrm{~N}_{6} \mathrm{O}$ : (\%): C, 69.10; $\mathrm{H}, 4.74 ; \mathrm{N}$, 21.98. Found: C, 69.48; H, 4.51; N, 21.67.

5-(4-(3-(3,4-Dimethoxyphenyl)-4,5-dihydro-1H-pyrazol-5-yl) phenoxy)-1-phenyl-1H-tetrazole (6b)

Yield 76\%; mp 162-164 ${ }^{\circ} \mathrm{C}$; IR; $3347(\mathrm{NH}), 1604(\mathrm{C}=\mathrm{N})$, 1141 (C-O). ${ }^{1} \mathrm{H}$ NMR; 2.75 (dd, 1H, $\mathrm{C}_{4}$ pyrazole, $J=10.8, J=$ 8.7), $3.31\left(\mathrm{~s}, 6 \mathrm{H}, 2 \mathrm{OC}_{3}\right), 3.84\left(\mathrm{dd}, 1 \mathrm{H}, \mathrm{C}_{4}\right.$ pyrazole, $J=8.7, J=$ 4.4), 4.70 (dd, $1 \mathrm{H}, \mathrm{C}_{5}$ pyrazole, $\left.J=10.8, J=4.4\right), 6.70-7.77$ (m, $12 \mathrm{H}, \mathrm{ArH}), 9.20$ (s, 1H, N-H). MS m/z (\%); $442.45\left(\mathrm{M}^{+}, 1.56\right)$, 440.45 (2.05), 328.14 (13.92), 118.13 (16.68), 55.03 (100), 43.11 (55.63). Anal. Calcd for $\mathrm{C}_{24} \mathrm{H}_{22} \mathrm{~N}_{6} \mathrm{O}_{3}(\%)$ : C, 65.15; H, 5.01; N, 18.99. Found: C, 65.53; H, 5.31; N, 18.67. 
5-(4-(3-(3,4-Dimethoxyphenyl)-4,5-dihydro-1H-pyrazol-5-yl)-2methoxyphenoxy)-1-phenyl-1H-tetrazole (6c)

Yield 61\%; mp 150-152 ${ }^{\circ} \mathrm{C}$; IR; 3347(NH), $1604(\mathrm{C}=\mathrm{N})$, $1141(\mathrm{C}-\mathrm{O}) .{ }^{1} \mathrm{H}$ NMR; $2.79\left(\mathrm{dd}, 1 \mathrm{H}, \mathrm{C}_{4}\right.$ pyrazole, $J=18.1, J=$ 11.7), $3.12\left(\mathrm{dd}, 1 \mathrm{H}, \mathrm{C}_{4}\right.$ pyrazole, $\left.J=18.1, J=5.1\right), 3.38(\mathrm{~s}, 6 \mathrm{H}, 2$ $\left.\mathrm{OC}_{3}\right), 3.63\left(\mathrm{~s}, 6 \mathrm{H}, 2 \mathrm{OCH}_{3}\right), 4.87\left(\mathrm{dd}, 1 \mathrm{H}, \mathrm{C}_{5}\right.$ pyrazole, $J=11.7$, $J=5.1), 6.73-7.76(\mathrm{~m}, 11 \mathrm{H}, \mathrm{ArH}), 9.18\left(\mathrm{~s}, 1 \mathrm{H}, \mathrm{D}_{2} \mathrm{O}\right.$ exchangeable, $\mathrm{N}-\mathrm{H}) . \mathrm{MS} \mathrm{m} / \mathrm{z}(\%) ; 472.00\left(\mathrm{M}^{+}, 2.47\right), 470.00$ (1.45), 264.25 (100), 152.13 (41.72), 115.13 (55.63), 71.07 (98.41), 57.03 (76.17). Anal. Calcd for $\mathrm{C}_{25} \mathrm{H}_{24} \mathrm{~N}_{6} \mathrm{O}_{4}(\%): \mathrm{C}, 63.55 ; \mathrm{H}, 5.12 ; \mathrm{N}$, 17.79 Found: C, 63.43; H, 5.44; N, 18.07 .
General Procedure for Synthesis of E-1-(aryl or heteroaryl)-3-(3methoxy-4-((1-phenyl-1H-tetrazol-5-yl)oxy)phenyl)prop-2-en-1ones (8a-g) (Scheme 2)

3-Methoxy-4-((1-phenyl-1H-tetrazol-5-yl)oxy) benzaldehyde (7) (2.96 g, $10 \mathrm{mmol})$ was added, while stirring, to a solution of the appropriate ketone $(1 \mathrm{c}-\mathbf{i})(10 \mathrm{mmol})$ in ethanolic $\mathrm{NaOH}$ solution $(2.5 \%, 10 \mathrm{~mL})$. Stirring was continued at room temperature for 2 hours. The reaction mixture was then acidified with dilute $\mathrm{HCl}$ and the separated product was collected by filtration, washed with water, dried and crystallized from ethanol, to give the required chalcone derivatives $\mathbf{8 a - g}$.

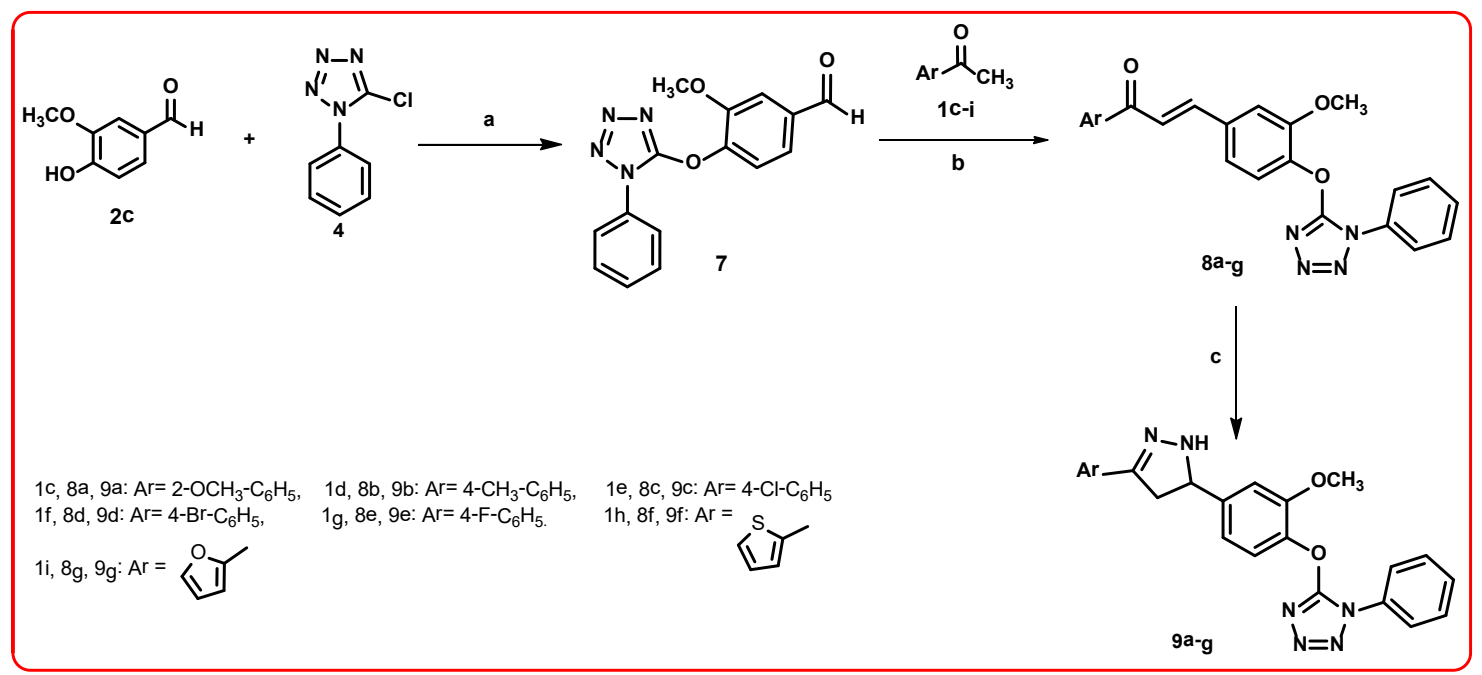

Scheme 2: Reagents and conditions: (a) $\mathrm{K}_{2} \mathrm{CO}_{3}$, DMF, $24 \mathrm{~h}$, RT. (b) $2.5 \% \mathrm{NaOH}, \mathrm{C}_{2} \mathrm{H}_{5} \mathrm{OH}, 2 \mathrm{~h}$ (c) $\mathrm{NH}_{2} \mathrm{NH}_{2} . \mathrm{H}_{2} \mathrm{O}, \mathrm{C}_{2} \mathrm{H}_{5} \mathrm{OH}$, reflux, 10-15 h.

3-(3-Methoxy-4-((1-phenyl-1H-tetrazol-5-yl)oxy)phenyl)-1-(2methoxyphenyl)prop-2-en-1-one (8a)

Yield $65 \%$; mp $102-103^{\circ} \mathrm{C}$; IR; $1660(\mathrm{C}=\mathrm{O}), 1596$ $(\mathrm{C}=\mathrm{C}), 1146(\mathrm{C}-\mathrm{O}) .{ }^{1} \mathrm{H} \mathrm{NMR} ; 3.78\left(\mathrm{~s}, 3 \mathrm{H}, \mathrm{OCH}_{3}\right), 3.94(\mathrm{~s}, 3 \mathrm{H}$, $\left.\mathrm{OCH}_{3}\right), 7.27(\mathrm{~d}, 1 \mathrm{H}, \mathrm{CO}-\mathrm{CH}=\mathrm{CH}, J=15), 7.43-7.54(\mathrm{~m}, 3 \mathrm{H}$, $\mathrm{ArH}), 7.64$ (d, 1H, CO-CH= $\underline{\mathrm{H}}, J=15), 7.78-8.02$ (m, 9H, ArH). Anal. Calcd. for $\mathrm{C}_{24} \mathrm{H}_{20} \mathrm{~N}_{4} \mathrm{O}_{4}(\%)$ : C, 67.28; H, 4.71; N, 13.08. Found: C, 67.46; H, 5.09; N, 13.37 .

3-(3-Methoxy-4-((1-phenyl-1H-tetrazol-5-yl)oxy)phenyl)-1-(ptolyl)prop-2-en-1-one (8b)

Yield $69 \%$; mp $142-144^{\circ} \mathrm{C}$; IR; $1665(\mathrm{C}=\mathrm{O}), 1606(\mathrm{C}=\mathrm{C})$, 1162 (C-O). ${ }^{1} \mathrm{HNMR} ; 2.33$ (s, 3H, $\mathrm{CH}_{3}$ ), 3.87 (s, 3H, $\mathrm{OCH}_{3}$ ), 7.237.59 (m, 4H, ArH), $7.64(\mathrm{~d}, 1 \mathrm{H}, \mathrm{CO}-\mathrm{C} \underline{\mathrm{H}}=\mathrm{CH}, J=16), 7.68-7.87$ $(\mathrm{m}, 6 \mathrm{H}, \mathrm{ArH}), 8.01(\mathrm{~d}, 1 \mathrm{H}, \mathrm{CO}-\mathrm{CH}=\mathrm{C} \underline{\mathrm{H}}, J=16), 8.10-8.12(\mathrm{~m}$, $2 \mathrm{H}, \mathrm{ArH})$. Anal. Calcd for $\mathrm{C}_{24} \mathrm{H}_{20} \mathrm{~N}_{4} \mathrm{O}_{3}(\%): \mathrm{C}, 69.89 ; \mathrm{H}, 4.89 ; \mathrm{N}$, 13.58. Found: C, 70.13; H, 4.54; N, 13.78 .

1-(4-Chlorophenyl)-3-(3-methoxy-4-((1-phenyl-1H-tetrazol-5yl) oxy)phenyl)prop-2-en-1-one (8c)

Yield $82 \%$; mp $183-185^{\circ} \mathrm{C}$; IR; $1666(\mathrm{C}=\mathrm{O}), 1602$ $(\mathrm{C}=\mathrm{C}), 1456$, $(\mathrm{C}-\mathrm{O}) .{ }^{1} \mathrm{H} \mathrm{NMR} ; 3.89\left(\mathrm{~s}, 3 \mathrm{H}, \mathrm{OCH}_{3}\right), 7.62(\mathrm{~d}$, $1 \mathrm{H}, \mathrm{CO}-\mathrm{C} \underline{\mathrm{H}}=\mathrm{CH}, J=16.5), 7.68-7.88(\mathrm{~m}, 9 \mathrm{H}, \mathrm{ArH}), 7.98$ $(\mathrm{d}, 1 \mathrm{H}, \mathrm{CO}-\mathrm{CH}=\mathrm{C} \underline{\mathrm{H}}, J=16.5), 8.02-8.23(\mathrm{~m}, 3 \mathrm{H}, \mathrm{ArH})$. ${ }^{13} \mathrm{C}$ NMR; $191.93(\mathrm{C}=\mathrm{O}), 159.05,156.64,150.32(\mathrm{O}-\underline{\mathrm{C}}-\mathrm{N})$, $146.17,143.51$ (C-Cl), 138.07, 138.21, 136.12, 130.49, 130.05
(2C), 129.97, 129.73 (2C), 128.91, 128.75 (2C), 123.65 (2C), $122.65,121.02,56.42\left(\mathrm{OCH}_{3}\right)$. Anal. Calcd for $\mathrm{C}_{23} \mathrm{H}_{17} \mathrm{ClN}_{4} \mathrm{O}_{3}$ (\%): C, 63.82; H, 3.96; N, 12.94. Found: C, 63.51; H, 4.20; $\mathrm{N}, 13.25$.

1-(4-Bromophenyl)-3-(3-methoxy-4-((1-phenyl-1H-tetrazol-5-yl) oxy)phenyl)prop-2-en-1-one (8d)

Yield 62\%; mp 190-192 ${ }^{\circ} \mathrm{C}$; IR; $1664(\mathrm{C}=\mathrm{O}), 1602$ $(\mathrm{C}=\mathrm{C}), 1164(\mathrm{C}-\mathrm{O}) .{ }^{1} \mathrm{H}$ NMR; $3.81\left(\mathrm{~s}, 3 \mathrm{H}, \mathrm{OCH}_{3}\right), 7.18-7.55(\mathrm{~m}$, $9 \mathrm{H}, \mathrm{ArH}), 7.57$ (d, 1H, CO-C $\underline{\mathrm{H}}=\mathrm{CH}, J=15.5), 7.68-7.78(\mathrm{~m}, 3 \mathrm{H}$, $\mathrm{ArH}), 7.81(\mathrm{~d}, 1 \mathrm{H}, \mathrm{CO}-\mathrm{CH}=\mathrm{CH}, J=15.5) .{ }^{13} \mathrm{C} \mathrm{NMR} ; 189.12$ $(\mathrm{C}=\mathrm{O}), 159.67,156.74,150.78,144.06,143.99,141.45,136.73$, 133.23 (2C), 132.15, 132.02, 130.06, 129.73, 129.40, 129.29 (2C), 128.11, 122.49 (2C), 122.06, 121.52, $56.35\left(\mathrm{OCH}_{3}\right)$. Anal. Calcd for $\mathrm{C}_{23} \mathrm{H}_{17} \mathrm{BrN}_{4} \mathrm{O}_{3}(\%)$ : C, 57.88; H, 3.59; N,11.74. Found: C, 58.12; H, 4.25; N, 11.45 .

1-(4-Fluorophenyl)-3-(3-methoxy-4-((1-phenyl-1H-tetrazol-5-yl) oxy)phenyl)prop-2-en-1-one (8e)

Yield $78 \%$; mp $155-157^{\circ} \mathrm{C}$; IR; $1676(\mathrm{C}=\mathrm{O}), 1601$ $(\mathrm{C}=\mathrm{C}), 1154(\mathrm{C}-\mathrm{O}) .{ }^{1} \mathrm{H}$ NMR; $3.88\left(\mathrm{~s}, 3 \mathrm{H}, \mathrm{OCH}_{3}\right), 7.11(\mathrm{~d}, 1 \mathrm{H}$, $\mathrm{CO}-\mathrm{C} \underline{\mathrm{H}}=\mathrm{CH}, J=15), 7.24-7.87(\mathrm{~m}, 9 \mathrm{H}, \mathrm{ArH}), 8.04(\mathrm{~d}, 1 \mathrm{H}$, $\mathrm{CO}-\mathrm{CH}=\mathrm{CH}, J=15), 8.09-8.31$ (m, 3H, ArH). Anal. Calcd for $\mathrm{C}_{23} \mathrm{H}_{17} \mathrm{FN}_{4} \mathrm{O}_{3}$ (\%): C, 66.34; H, 4.12; N, 13.45. Found: C, 66.04; $\mathrm{H}, 4.45 ; \mathrm{N}, 13.15$. 
3-(3-Methoxy-4-((1-phenyl-1H-tetrazol-5-yl)oxy)phenyl)-1-

(thiophen-2-yl)prop-2-en-1-one (8f)

Yield $68 \%$; mp $110-112^{\circ} \mathrm{C}$; IR; $1652(\mathrm{C}=\mathrm{O}), 1599$ $(\mathrm{C}=\mathrm{C}), 1115(\mathrm{C}-\mathrm{O}) .{ }^{1} \mathrm{H}$ NMR; $3.90\left(\mathrm{~s}, 3 \mathrm{H}, \mathrm{OCH}_{3}\right), 7.34$ (d, 1H, $\mathrm{CO}-\mathrm{CH}=\mathrm{CH}, J=16.5), 7.37-7.62(\mathrm{~m}, 8 \mathrm{H}, \mathrm{ArH}), 7.80(\mathrm{~d}, 1 \mathrm{H}$, $\mathrm{CO}-\mathrm{CH}=\mathrm{C} \underline{\mathrm{H}}, J=16.5), 7.83-7.90(\mathrm{~m}, 11 \mathrm{H}, \mathrm{ArH}) .{ }^{13} \mathrm{C} \mathrm{NMR}$; $189.98(\mathrm{C}=\mathrm{O}), 159.73,151.21,150.75,146.82,143.88,143.53$, $139.42,135.77,134.85,133.26,132.02,129.74,129.41$ (2C), $128.42,123.14$ (2C), 122.19, 121.54, $56.36\left(\mathrm{O}_{\underline{C}}{ }_{3}\right)$. Anal. Calcd for $\mathrm{C}_{21} \mathrm{H}_{16} \mathrm{~N}_{4} \mathrm{O}_{3} \mathrm{~S}(\%)$ : C, 62.36; H, 3.99; N, 13.85; S, 7.89. Found: C, 62.68; H, 4.31; N, 14.23; S, 7.49.

1-(Furan-2-yl)-3-(3-methoxy-4-((1-phenyl-1H-tetrazol-5-yl)oxy) phenyl)prop-2-en-1-one (8g)

Yield 73\%; mp 94-95 C; IR; $1657(\mathrm{C}=\mathrm{O}), 1603(\mathrm{C}=\mathrm{C})$, 1159 (C-O). ${ }^{1} \mathrm{H}$ NMR; 3.81 (s, 3H, $\mathrm{OCH}_{3}$ ), 7.19-7.29 (m, 4H, ArH), $7.35(\mathrm{~d}, 1 \mathrm{H}, \mathrm{CO}-\mathrm{CH}=\mathrm{CH}, J=16), 7.46(\mathrm{~d}, 1 \mathrm{H}, \mathrm{CO}-\mathrm{CH}=\mathrm{C} \underline{\mathrm{H}}, J=$ 16), 7.48-7.92 (m, 7H, ArH). ${ }^{13} \mathrm{C}$ NMR; $190.62(\mathrm{C}=\mathrm{O}), 159.31$, $153.62,151.21,152.84,146.83,146.64,144.53,142.68,133.13$, 129.76, 129.51 (2C), 129.38, 124.83 (2C), 122.00, 121.55, 117.75, 112.69, $56.35\left(\mathrm{OCH}_{3}\right)$. Anal. Calcd for $\mathrm{C}_{21} \mathrm{H}_{16} \mathrm{~N}_{4} \mathrm{O}_{4}(\%)$ : C, 64.94; H, 4.15; N,14.43. Found: C, 65.18; H, 4.31; N, 14.23.

General Procedure for Synthesis of 5-(2-methoxy-4-(3-aryl or heteroaryl-4,5-dihydro-1H-pyrazol-5-yl)phenoxy)-1-phenyl-1Htetrazoles (9a-g) (Scheme 2)

A mixture of a chalcone derivative (8a-g) $(10 \mathrm{mmol})$ and hydrazine monohydrate $(95 \%)(1.2 \mathrm{~mL}, 20 \mathrm{mmol})$ in ethanol $(30$ $\mathrm{mL}$ ) was heated at reflux temperature for 10-15 hours. The reaction mixture was cooled, poured onto crushed ice and the obtained solid product was collected by filtration, washed with water, dried and crystallized from ethanol, to furnish the pyrazolines $9 \mathbf{a - g}$.

5-(2-Methoxy-4-(3-(2-methoxyphenyl)-4,5-dihydro-1H-pyrazol5-yl)phenoxy)-1-phenyl-1H-tetrazole (9a)

Yield 70\%; mp 201-202 ${ }^{\circ} \mathrm{C}$; IR; $3347(\mathrm{NH}), 1574(\mathrm{C}=\mathrm{N})$, 1174 (C-O). ${ }^{1} \mathrm{H}$ NMR; 2.45-2.51 (m, 1H, C 4 pyrazole), 3.30-3.40 (m, 2H, C 4 pyrazole), $3.75\left(\mathrm{~s}, 6 \mathrm{H}, 2 \mathrm{OCH}_{3}\right), 4.56-4.62(\mathrm{~m}, 1 \mathrm{H}$, $\mathrm{C}_{5}$ pyrazole), 6.70-7.76 (m, 12H, ArH), $9.21(\mathrm{~s}, 1 \mathrm{H}, \mathrm{N}-\mathrm{H}) . \mathrm{MS}$ $\mathrm{m} / \mathrm{z}(\%) ; 442.16\left(\mathrm{M}^{+}, 2.35\right), 440.16$ (1.11), 256.32 (7.78), 152.13 (13.90), 98.11 (100), 83.09 (23.35), 55.03 (80.62). Anal. Calcd for $\mathrm{C}_{24} \mathrm{H}_{22} \mathrm{~N}_{6} \mathrm{O}_{3}(\%): \mathrm{C}, 65.15 ; \mathrm{H}, 5.01 ; \mathrm{N}, 18.99$. Found: C, 65.43; H, $5.34 ; \mathrm{N}, 18.77$.

5-(2-Methoxy-4-(3-(p-tolyl)-4,5-dihydro-1H-pyrazol-5-yl) phenoxy)-1-phenyl-1H-tetrazole (9b)

Yield $66 \%$; mp $122-124^{\circ} \mathrm{C}$; IR; $3350(\mathrm{NH}), 1604$ $(\mathrm{C}=\mathrm{N}), 1177(\mathrm{C}-\mathrm{O}) .{ }^{1} \mathrm{H}$ NMR; $2.27\left(\mathrm{~s}, 3 \mathrm{H}, \mathrm{CH}_{3}\right), 2.35-2.41(\mathrm{~m}$, $1 \mathrm{H}, \mathrm{C}_{4}$ pyrazole $), 3.31-3.40\left(\mathrm{~m}, 1 \mathrm{H}, \mathrm{C}_{4}\right.$ pyrazole $), 3.86(\mathrm{~s}, 3 \mathrm{H}$, $\left.\mathrm{OCH}_{3}\right), 4.55-4.61\left(\mathrm{~m}, 1 \mathrm{H}, \mathrm{C}_{5}\right.$ pyrazole $), 6.73-7.77(\mathrm{~m}, 12 \mathrm{H}$, ArH), 9.23 (s, 1H, N-H). ${ }^{13} \mathrm{C}$ NMR; 159.34, 151.83, 149.30, $147.94,146.13,140.18,137.89,134.17,129.72$ (2C), 129.53, 129.31 (2C), 125.82 (2C), 125.44 (2C), 121.94, 117.74, 111.23, $56.14\left(\mathrm{OC}_{3}\right), 56.02,41.12\left(\underline{\mathrm{CH}}_{2}\right), 21.35\left(\underline{\mathrm{CH}}_{3}\right) . \mathrm{MS}$ $\mathrm{m} / \mathrm{z}(\%) ; 426.19\left(\mathrm{M}^{+}, 1.45\right), 424.19$ (2.22), 135.01 (19.46), 111.19 (62.83), 71.07 (35.03), 57.03 (100), 51.01 (30.02).
Anal. Calcd for $\mathrm{C}_{24} \mathrm{H}_{22} \mathrm{~N}_{6} \mathrm{O}$ (\%): C, 67.59; H, 5.20; N, 19.71. Found: C, 67.43; H, 5.34; N, 19.87 .

5-(4-(3-(4-Chlorophenyl)-4,5-dihydro-1H-pyrazol-5-yl)-2methoxyphenoxy)-1-phenyl-1H-tetrazole (9c)

Yield 69\%; mp 194-196 ${ }^{\circ} \mathrm{C}$; IR; 3347 (NH), 1574 $(\mathrm{C}=\mathrm{N}), 1174(\mathrm{C}-\mathrm{O}) .{ }^{1} \mathrm{H}$ NMR; 2.60-2.69 (m, 1H, $\mathrm{C}_{4}$ pyrazole), 3.32-3.42 (m, 1H, $\mathrm{C}_{4}$ pyrazole), $3.91\left(\mathrm{~s}, 3 \mathrm{H}, \mathrm{OCH}_{3}\right), 4.56-4.62(\mathrm{~m}$, $1 \mathrm{H}, \mathrm{C}_{5}$ pyrazole), 6.71-8.18 (m, 12H, ArH), $9.22(\mathrm{~s}, 1 \mathrm{H}, \mathrm{N}-\mathrm{H}) \cdot{ }^{13} \mathrm{C}$ NMR; 159.41, 153.73, 151.83, 149.30, 146.13, 140.18, 137.89, $134.17,129.72,129.53$ (2C), 129.31 (2C), 125.82 (2C), 124.34 (2C), 121.94, 117.74, 111.23, $56.14\left(\mathrm{OCH}_{3}\right), 56.02,41.12\left(\underline{\mathrm{CH}}_{2}\right)$. MS m/z (\%); $448.90\left(\mathrm{M}^{+}+2,1.11\right), 447.90\left(\mathrm{M}^{+}+1,2.78\right), 446.90$ $\left(\mathrm{M}^{+}, 3.34\right), 264.12$ (57.82), 117.00 (72.84), 109.12 (100). Anal. Calcd for $\mathrm{C}_{23} \mathrm{H}_{19} \mathrm{ClN}_{6} \mathrm{O}_{2}(\%)$ : C, 61.82; H, 4.29; N, 18.81. Found: C, 61.43; H, 4.34; N, 19.07 .

5-(4-(3-(4-Bromophenyl)-4,5-dihydro-1H-pyrazol-5-yl)-2methoxyphenoxy)-1-phenyl-1H-tetrazole (9d)

Yield 64\%; mp 184-185 ${ }^{\circ} \mathrm{C}$; IR; $3347(\mathrm{NH}), 1578(\mathrm{C}=\mathrm{N})$, 1175 (C-O). ${ }^{1} \mathrm{H}$ NMR; 2.52-2.60 (m, $1 \mathrm{H}, \mathrm{C}_{4}$ pyrazole), 3.34-3.39 $\left(\mathrm{m}, 1 \mathrm{H}, \mathrm{C}_{4}\right.$ pyrazole $), 3.79\left(\mathrm{~s}, 3 \mathrm{H}, \mathrm{OCH}_{3}\right), 4.32-4.40(\mathrm{~m}, 1 \mathrm{H}$, $\mathrm{C}_{5}$ pyrazole), 6.71-8.10 (m, 12H, ArH), $9.24(\mathrm{~s}, 1 \mathrm{H}, \mathrm{N}-\mathrm{H}) .{ }^{13} \mathrm{C}$ NMR; 159.32,151.82, 150.40, 148.47, 146.01, 140.17, 137.43, 130.90 (2C), 130.72, 129.30 (2C), 127.49 (2C), 127.39, 124.87 (2C), 121.94, 117.73, 112.23, $56.31\left(\mathrm{O}_{\underline{C}}{ }_{3}\right), 55.32,40.57\left(\underline{\mathrm{CH}}_{2}\right)$. MS m/z (\%); $493.06\left(\mathrm{M}^{+}+2,2.82\right), 492.06\left(\mathrm{M}^{+}+1,1.67\right), 491.06$ $\left(\mathrm{M}^{+}, 2.53\right), 331.95$ (60.61), 253.08 (80.62), 145.01 (65.05), 55.03 (100). Anal. Calcd for $\mathrm{C}_{23} \mathrm{H}_{19} \mathrm{BrN}_{6} \mathrm{O}_{2}(\%)$ : C, 56.22; H, 3.90; N, 17.10. Found: C, 56.43; H, 4.34; N, 17.07 .

5-(4-(3-(4-Fluorophenyl)-4,5-dihydro-1H-pyrazol-5-yl)-2methoxyphenoxy)-1-phenyl-1H-tetrazole (9e)

Yield 77\%; mp 114-115 ${ }^{\circ} \mathrm{C}$; IR; $3347(\mathrm{NH}), 1603(\mathrm{C}=\mathrm{N})$, 1141 (C-O). ${ }^{1} \mathrm{H}$ NMR; 2.61-2.70 (m, 1H, C 4 pyrazole), 3.40-3.48 $\left(\mathrm{m}, 1 \mathrm{H}, \mathrm{C}_{4}\right.$ pyrazole $), 3.79\left(\mathrm{~s}, 3 \mathrm{H}, \mathrm{OCH}_{3}\right), 4.70-4.81\left(\mathrm{~m}, 1 \mathrm{H}, \mathrm{C}_{5}\right.$ pyrazole), 6.71-7.89 (m, 12H, ArH), 9.20 (s, 1H, N-H). ${ }^{13} \mathrm{CNMR}$; 164.59 (C-F), 159.32, 151.82, 150.42, 148.46, 146.15, 140.16, 131.84, 129.30 (2C), 127.84, 127.78 (2C), 124.43 (2C), 121.94, $117.73,115.80,115.68,111.23,56.30\left(\mathrm{O}_{\mathbf{C H}}\right), 56.02,41.09$ $\left(\mathrm{CH}_{2}\right)$. Anal. Calcd for $\mathrm{C}_{23} \mathrm{H}_{19} \mathrm{FN}_{6} \mathrm{O}_{2}(\%)$ : C, 64.18; H, 4.45; N, 19.52. Found: C, 64.43; H, 4.34; N, 19.17.

5-(2-Methoxy-4-(3-(thiophen-2-yl)-4,5-dihydro-1H-pyrazol-5-yl) phenoxy)-1-phenyl-1H-tetrazole (9f)

Yield 62\%; mp 134-135 C; IR; $3348(\mathrm{NH}), 1604(\mathrm{C}=\mathrm{N})$ 1112 (C-O). ${ }^{1} \mathrm{H}$ NMR; 2.48-2.52 (m, 1H, $\mathrm{C}_{4}$ pyrazole), 3.35-3.41 $\left(\mathrm{m}, 1 \mathrm{H}, \mathrm{C}_{4}\right.$ pyrazole), $3.88\left(\mathrm{~s}, 3 \mathrm{H}, \mathrm{OCH}_{3}\right), 4.70-4.81\left(\mathrm{~m}, 1 \mathrm{H}, \mathrm{C}_{5}\right.$ pyrazole), 6.69-8.03 (m, 11H, ArH), 9.23 (s, 1H, N-H). ${ }^{13} \mathrm{C}$ NMR; $159.24,151.82,150.26,148.45,146.19,140.16,129.31,129.16$ (2C), 127.95, 126.68, 126.55, 126.47, 124.59 (2C), 121.95, $\left.117.73,111.22,56.30\left(\mathrm{O}_{\underline{C}}\right)_{3}\right), 56.03,41.93\left(\underline{\mathrm{CH}}_{2}\right) . \mathrm{MS} \mathrm{m} / \mathrm{z}(\%)$; $420.11\left(\mathrm{M}^{+}+2,4.32\right), 418.11\left(\mathrm{M}^{+}, 2.23\right), 258.07$ (100), 135.07 (26.69), 115.07 (22.24). Anal. Calcd for $\mathrm{C}_{21} \mathrm{H}_{18} \mathrm{~N}_{6} \mathrm{O}_{2} \mathrm{~S}(\%)$ : C, 60.27; H, 4.34; N, 20.08; S, 7.66. Found: C, 60.43; H, 4.35; N, $19.78 ; \mathrm{S}, 8.02$. 
5-(4-(3-(Furan-2-yl)-4,5-dihydro-1H-pyrazol-5-yl)-2-

methoxyphenoxy)-1-phenyl-1H-tetrazole (9g)

Yield 61\%; mp 193-195 C; IR; $3347(\mathrm{NH}) 1604(\mathrm{C}=\mathrm{N})$, 1143 (C-O). ${ }^{1} \mathrm{H}$ NMR; 2.50-2.58 ( $\mathrm{m}, 1 \mathrm{H}, \mathrm{C}_{4}$ pyrazole), 3.36-3.42 (m, 2H, $\mathrm{C}_{4}$ pyrazole), $3.90\left(\mathrm{~s}, 3 \mathrm{H}, \mathrm{OCH}_{3}\right), 4.71-4.76\left(\mathrm{~m}, 1 \mathrm{H}, \mathrm{C}_{5}\right.$ pyrazole), 6.59-7.92 (m, 11H, ArH), 9.19 (s, 1H, N-H). MS m/z (\%); $402.40\left(\mathrm{M}^{+}, 2.76\right), 400.40$ (2.22), 281.03 (15.01), 207.02 (47.26), 111.13 (100), 67.06 (85.07), 53.08 (52.26). Anal. Calcd for $\mathrm{C}_{21} \mathrm{H}_{18} \mathrm{~N}_{6} \mathrm{O}_{3}(\%)$ : C, 62.68; $\mathrm{H}, 4.51 ; \mathrm{N}, 20.88$. Found: $\mathrm{C}, 62.43$; $\mathrm{H}, 4.34 ; \mathrm{N}, 19.07$.
General Procedure for Synthesis of E-1-(4-((1-phenyl-1Htetrazol-5-yl)oxy)phenyl)-3-(un) substituted phenylprop-2-en-1ones (11a-i) (Scheme 3)

Compound 4-((1-Phenyl-1H-tetrazol-5-yl)oxy) acetophenone (10) (2.8 g, $10 \mathrm{mmol})$ was added, while stirring, to the appropriate benzaldehyde (2d-i) $(10 \mathrm{mmol})$ in ethanolic $\mathrm{NaOH}$ solution $(2.5 \%, 10 \mathrm{~mL})$. Stirring was continued for additional 2 hours. The reaction mixture was then acidified with dilute $\mathrm{HCl}$ and the separated compounds were collected by filtration, washed with water, dried and crystallized from ethanol to give 11a-i.

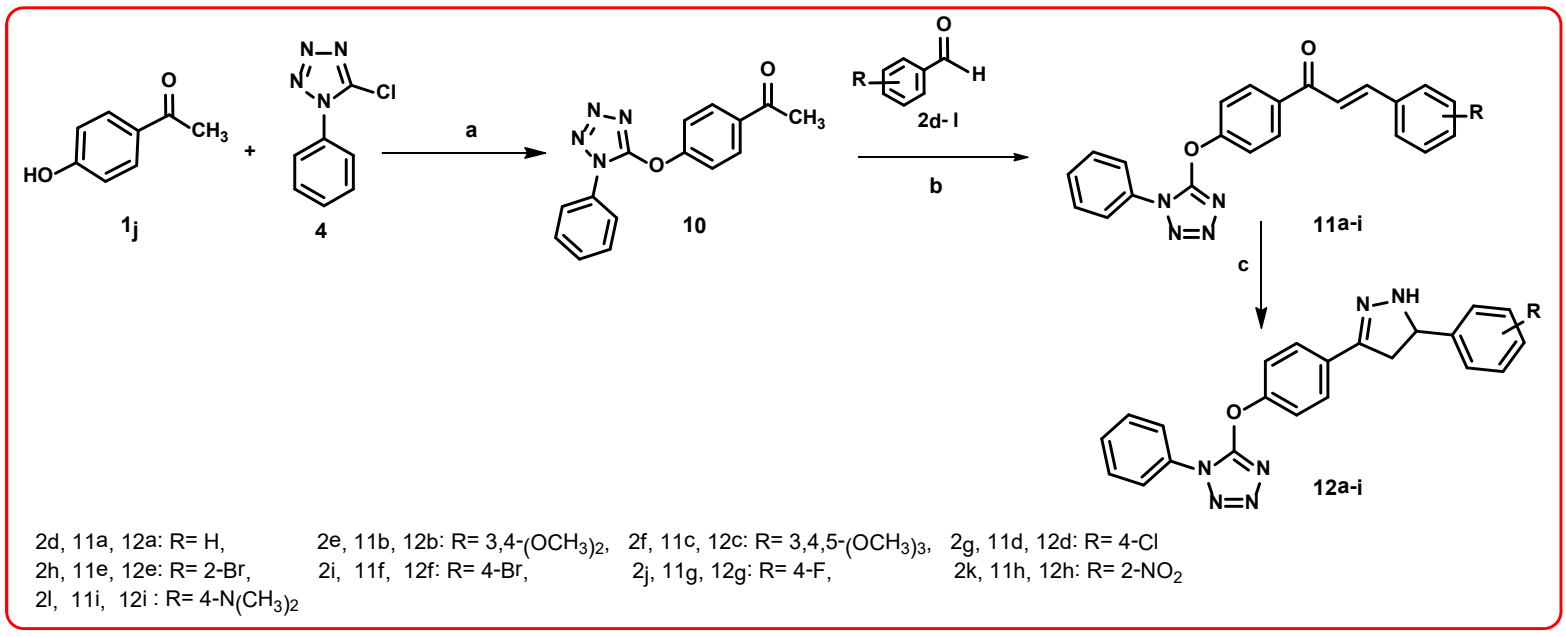

Scheme 3: Reagents and conditions: (a) $\mathrm{K}_{2} \mathrm{CO}_{3}$, DMF, 24 h, RT. (b) $2.5 \% \mathrm{NaOH}, \mathrm{C}_{2} \mathrm{H}_{5} \mathrm{OH}, 2 \mathrm{~h}$ (c) $\mathrm{NH}_{2} \mathrm{NH}_{2} . \mathrm{H}_{2} \mathrm{O}, \mathrm{C}_{2} \mathrm{H}_{5} \mathrm{OH}$, reflux, $10-15 \mathrm{~h}$.

E-3-Phenyl-1-(4-((1-phenyl-1H-tetrazol-5-yl)oxy)phenyl)prop-2en-1-one (11a)

Yield $71 \%$; mp 103-105 ${ }^{\circ}$; IR; $1679(\mathrm{C}=\mathrm{O}), 1598$ $(\mathrm{C}=\mathrm{C}), 1163(\mathrm{C}-\mathrm{O}) .{ }^{1} \mathrm{H}$ NMR; 7.48-7.69 (m, 11H, ArH), $7.73(\mathrm{~d}$, $1 \mathrm{H}, \mathrm{CO}-\mathrm{C} \underline{\mathrm{H}}=\mathrm{CH}, J=13.5), 7.75-7.82(\mathrm{~m}, 3 \mathrm{H}, \mathrm{ArH}), 7.89(\mathrm{~d}, 1 \mathrm{H}$, $\mathrm{CO}-\mathrm{CH}=\mathrm{CH}, J=13.5) .{ }^{13} \mathrm{C}$ NMR $\delta(\mathrm{ppm}) ; 187.89(\mathrm{C}=\mathrm{O}), 159.92$, $159.01,156.48,144.34,134.59,132.76,131.49,130.78,129.92$, $129.83,129.72,129.29,128.95,128.90,128.76(2 \mathrm{C}), 123.31(2 \mathrm{C})$, 122.45 (2C), 121.86. Calcd for $\mathrm{C}_{22} \mathrm{H}_{16} \mathrm{~N}_{4} \mathrm{O}_{2}(\%)$ : C, 71.73; H, 4.38; N, 15.21. Found: C, 71.53; H, 4.29; N, 15.54.

\section{E-3-(3,4-Dimethoxyphenyl)-1-(4-((1-phenyl-1H-tetrazol-5-yl) oxy)phenyl) prop-2-en-1-one (11b)}

Yield 69\%; mp 76-78 $\mathrm{C}$; IR; $1680(\mathrm{C}=\mathrm{O}), 1595(\mathrm{C}=\mathrm{C})$, 1160, (C-O). ${ }^{1} \mathrm{H}$ NMR; 3.88 (s, 6H, 2OCH $), 6.95-7.69$ (m, 12H, $\mathrm{ArH}), 7.74(\mathrm{~d}, 1 \mathrm{H}, \mathrm{CO}-\mathrm{C} \underline{\mathrm{H}}=\mathrm{CH}, J=13), 8.02(\mathrm{~d}, 1 \mathrm{H}, \mathrm{CO}-\mathrm{CH}=\mathrm{C} \underline{\mathrm{H}}$, $J=13) .{ }^{13} \mathrm{C}$ NMR; $190.84(\mathrm{C}=\mathrm{O}), 160.05,158.67,154.48,149.59$, $149.29,145.56,133.41,130.92,130.59,129.81,129.71,129.52$, 126.78, 123.40, 122.34 (2C), 121.51, 119.26 (2C), 110.49, 110.24, $55.96\left(2 \mathrm{OCH}_{3}\right)$. Anal. Calcd. for $\mathrm{C}_{24} \mathrm{H}_{20} \mathrm{~N}_{4} \mathrm{O}_{4}(\%): \mathrm{C}, 67.28 ; \mathrm{H}$, $4.71 ; \mathrm{N}, 13.08$. Found: C, 67.58; H, 4.98; N, 13.38 .

\section{E-3-(3,4,5-Trimethoxyphenyl)-1-(4-((1-phenyl-1H-tetrazol-5-yl) oxy)phenyl) prop-2-en-1-one (11c)}

Yield $61 \%$; mp 80-82 ${ }^{\circ} \mathrm{C} ;{ }^{1} \mathrm{H}$ NMR; 3.79 (s, 3H, $\mathrm{OCH}_{3}$ ), $3.88\left(\mathrm{~s}, 6 \mathrm{H}, 2 \mathrm{OCH}_{3}\right), 7.55(\mathrm{~d}, 1 \mathrm{H}, \mathrm{CO}-\mathrm{C} \underline{\mathrm{H}}=\mathrm{CH}, J=14), 7.61-$ $8.12(\mathrm{~m}, 11 \mathrm{H}, \mathrm{ArH}), 8.32(\mathrm{~d}, 1 \mathrm{H}, \mathrm{CO}-\mathrm{CH}=\mathrm{CH}, J=14) .{ }^{13} \mathrm{C}$ NMR; $191.83(\mathrm{C}=\mathrm{O}), 159.92,159.05,156.42,153.31,153.09$,
$144.88,139.87,132.76,130.73,130.40,129.92,129.83,129.73$, $129.29,123.31$ (2C), 122.45 (2C), 121.02, 106.72, 106.67, $56.13\left(\mathrm{OCH}_{3}\right), 56.03\left(\mathrm{OCH}_{3}\right), 55.71\left(\mathrm{OCH}_{3}\right)$. Anal. Calcd. for $\mathrm{C}_{25} \mathrm{H}_{22} \mathrm{~N}_{4} \mathrm{O}_{5}(\%)$ : C, 65.49; H, 4.84; N, 12.22. Found: C, 65.05; $\mathrm{H}, 4.68 ; \mathrm{N}, 12.50$.

\section{E-3-(4-Chlorophenyl)-1-(4-((1-phenyl-1H-tetrazol-5-yl)oxy) phenyl)prop-2-en-1-one (11d)}

Yield 85\%; mp $105-107^{\circ} \mathrm{C}$; IR; $1662(\mathrm{C}=\mathrm{O}), 1603$ $(\mathrm{C}=\mathrm{C}), 1166(\mathrm{C}-\mathrm{O}) .{ }^{1} \mathrm{H}$ NMR; 7.24-7.89 (m, 11H, ArH), 7.96 $(\mathrm{d}, 1 \mathrm{H}, \mathrm{CO}-\mathrm{C} \underline{\mathrm{H}}=\mathrm{CH}, J=14), 7.99-8.04(\mathrm{~m}, 2 \mathrm{H}, \mathrm{ArH}), 8.32(\mathrm{~d}$, $1 \mathrm{H}, \mathrm{CO}-\mathrm{CH}=\mathrm{C} \underline{\mathrm{H}}, J=14)$. Anal. Calcd. for $\mathrm{C}_{22} \mathrm{H}_{15} \mathrm{ClN}_{4} \mathrm{O}(\%)$ : $\mathrm{C}$, 65.06; H, 3.75; N, 13.91. Found: C, 65.36; H, 3.98; N, 13.78 .

\section{E-3-(2-Bromophenyl)-1-(4-((1-phenyl-1H-tetrazol-5-yl)oxy) phenyl)prop-2-en-1-one (11e)}

Yield $81 \%$; mp $168-170^{\circ} \mathrm{C}$; IR; $1665(\mathrm{C}=\mathrm{O}), 1603$ $(\mathrm{C}=\mathrm{C}), 1169,(\mathrm{C}-\mathrm{O}) .{ }^{1} \mathrm{H}$ NMR; $7.41(\mathrm{~d}, 1 \mathrm{H}, \mathrm{CO}-\mathrm{CH}=\mathrm{CH}, J=14)$, 7.49-8.03 (m, 11H, ArH), 8.22 (d, 1H, CO-CH=CH, $J=14), 8.33-$ 8.35 (m, 2H, ArH). Anal. Calcd for $\mathrm{C}_{22} \mathrm{H}_{15} \mathrm{BrN}_{4} \mathrm{O}_{2}(\%)$ : C, 59.08; H, 3.38; N, 12.53. Found: C, 59.36; H, 3.58; N, 12.84 .

\section{E-3-(4-Bromophenyl)-1-(4-((1-phenyl-1H-tetrazol-5-yl)oxy) phenyl)prop-2-en-1-one (11f)}

Yield 77\%; mp 92-94 ${ }^{\circ} \mathrm{C}$; IR; $1664(\mathrm{C}=\mathrm{O}), 1602(\mathrm{C}=\mathrm{C})$, 1166, (C-O). ${ }^{1} \mathrm{H}$ NMR; 7.18-7.65 (m, 6H, ArH), 7.69 (d, 1H, $\mathrm{CO}-\mathrm{CH}=\mathrm{CH}, J=12.2), 7.73-7.89(\mathrm{~m}, 4 \mathrm{H}, \mathrm{ArH}), 7.98(\mathrm{~d}, 1 \mathrm{H}$, $\mathrm{CO}-\mathrm{CH}=\mathrm{CH}, J=12.2), 8.02-8.34$ (m, 3H, ArH). Anal. Calcd. for 
$\mathrm{C}_{22} \mathrm{H}_{15} \mathrm{BrN}_{4} \mathrm{O}_{2}(\%)$ : C, 59.08; H, 3.38; N,12.53. Found: C, 59.36; $\mathrm{H}, 3.58 ; \mathrm{N}, 12.84$.

\section{E-3-(4-Fluorophenyl)-1-(4-((1-phenyl-1H-tetrazol-5-yl)oxy)}

phenyl)prop-2-en-1-one (11g)

Yield 89\%; mp 93-95 ${ }^{\circ}$; IR; $1670(\mathrm{C}=\mathrm{O}), 1598(\mathrm{C}=\mathrm{C})$, 1163, (C-O). ${ }^{1} \mathrm{H}$ NMR; 6.96-7.26 (m, 2H, ArH), 7.33 (d, 1H, $\mathrm{CO}-\mathrm{C} \underline{\mathrm{H}}=\mathrm{CH}, J=16.5), 7.59-7.69(\mathrm{~m}, 4 \mathrm{H}, \mathrm{ArH}), 7.75(\mathrm{~d}, 1 \mathrm{H}$, $\mathrm{CO}-\mathrm{CH}=\mathrm{C} \underline{\mathrm{H}}, J=16.5), 7.81-8.34(\mathrm{~m}, 7 \mathrm{H}, \mathrm{ArH})$. Anal. Calcd. for $\mathrm{C}_{22} \mathrm{H}_{15} \mathrm{FN}_{4} \mathrm{O}_{2}(\%): \mathrm{C}, 68.39 ; \mathrm{H}, 3.91 ; \mathrm{N}, 14.51$. Found: $\mathrm{C}, 68.09 ; \mathrm{H}$, $3.56 ; \mathrm{N}, 14.12$.

\section{E-3-(2-Nitrophenyl)-1-(4-((1-phenyl-1H-tetrazol-5-yl)oxy) phenyl)prop-2-en-1-one (11h)}

Yield $79 \%$; mp $170-172^{\circ} \mathrm{C}$; IR; $1670(\mathrm{C}=\mathrm{O}), 1549$ $1340\left(\mathrm{NO}_{2}\right), 1602(\mathrm{C}=\mathrm{C}), 1168(\mathrm{C}-\mathrm{O}),{ }^{1} \mathrm{H} \mathrm{NMR} ; 7.42$ (d, 1H, $\mathrm{CO}-\mathrm{CH}=\mathrm{CH}, J=15), 7.51-8.24(\mathrm{~m}, 13 \mathrm{H}, \mathrm{ArH}), 8.33(\mathrm{~d}, 1 \mathrm{H}$, $\mathrm{CO}-\mathrm{CH}=\mathrm{CH}, J=15) .{ }^{13} \mathrm{C}$ NMR; $187.61(\mathrm{C}=\mathrm{O}), 159.32,158.91$, $156.57,141.45,135.15,133.79,132.19,130.86,130.37,129.85$, $129.75,128.75$ (2C), 127.98, 127.77, 124.65 (2C), 123.25, 121.25, 120.03, 119.81. Anal. Calcd for $\mathrm{C}_{22} \mathrm{H}_{15} \mathrm{~N}_{5} \mathrm{O}_{4}(\%)$ : C, 63.92; $\mathrm{H}$, 3.66; N, 16.94. Found: C, 64.09; H, 3.70; N, 17.12.

E-3-(4-(Dimethylamino)phenyl)-1-(4-((1-phenyl-1H-tetrazol-5yl)oxy)phenyl) prop-2-en-1-one (11i)

Yield 77\%; mp 68-70 ${ }^{\circ} \mathrm{C} ;{ }^{1} \mathrm{H}$ NMR; 3.43 (s, 6H, 2CH ), 7.55-7.73 (m, 6H, $\mathrm{ArH}), 7.75(\mathrm{~d}, 1 \mathrm{H}, \mathrm{CO}-\mathrm{CH}=\mathrm{CH}, J=15.3)$, $7.85-8.25$ (m, 5H, ArH), 8.33 (d, $1 \mathrm{H}, \mathrm{CO}-\mathrm{CH}=\mathrm{C} \underline{\mathrm{H}}, J=15.3) .{ }^{13} \mathrm{C}$ NMR; $187.71(\mathrm{C}=\mathrm{O}), 159.86,158.90,156.63,156.39,148.73$, $133.67,130.94,130.33,129.86,129.76,129.66,129.47,129.23$, 124.63, 123.25 (2C), 122.38, 120.05 (2C), 119.83 (2C), 39.94 $\left(\underline{\mathrm{CH}}_{3}\right), 39.78\left(\underline{\mathrm{CH}}_{3}\right)$. Anal. Calcd for $\mathrm{C}_{24} \mathrm{H}_{21} \mathrm{~N}_{5} \mathrm{O}_{2}(\%)$ : C, 70.06; H, 5.14; N, 17.02. Found: C, 70.36; H, 5.45; N, 17.32.

General Procedure for Synthesis of 5-(4-(5-(substituted phenyl)4,5-dihydro-1H-pyrazol-3-yl)phenoxy)-1-phenyl-1H-tetrazoles (12a-i) (Scheme 3)

A mixture of a chalcone derivative (11a-i) $(10 \mathrm{mmol})$ and hydrazine monohydrate $(95 \%)(1.2 \mathrm{~mL}, 20 \mathrm{mmol})$ in ethanol $(30 \mathrm{~mL})$ was heated at reflux temperature for 10-15 hours. The reaction mixture was cooled, poured onto crushed ice and the obtaining solid product was collected by filtration, washed with water and crystallized from ethanol to give the pyrazoline compounds 12a-i.

1-Phenyl-5-(4-(5-phenyl-4,5-dihydro-1H-pyrazol-3-yl)phenoxy)1H-tetrazole (12a)

Yield 61\%; mp 200-202 ${ }^{\circ} \mathrm{C}$; IR; 3347(NH), $1604(\mathrm{C}=\mathrm{N})$, 1141 (C-O). ${ }^{1} \mathrm{H}$ NMR; 2.51-2.60 (m, 1H, C 4 pyrazole), 3.34-3.40 (m, 1H, $\mathrm{C}_{4}$ pyrazole), 4.45-4.51 (m, $1 \mathrm{H}, \mathrm{C}_{5}$ pyrazole), 6.69-7.77 (m, 14H, ArH), 9.17 (s, 1H, N-H). MS m/z (\%); $382.25\left(\mathrm{M}^{+}, 1.67\right)$, 380.25 (1.12), 97.11 (19.46), 69.06 (38.92), 109.12 (100), 43.11 (41.72). Anal. Calcd. for $\mathrm{C}_{22} \mathrm{H}_{18} \mathrm{~N}_{6} \mathrm{O}(\%)$ : C, 69.10; $\mathrm{H}, 4.74 ; \mathrm{N}$, 21.98. Found: C, 69.43; H, 4.34; N, 22.07.

5-(4-(5-(3,4-Dimethoxyphenyl)-4,5-dihydro-1H-pyrazol-3-yl) phenoxy)-1-phenyl-1H-tetrazole (12b)

Yield 63\%; mp 204-206 ${ }^{\circ}$; IR; $3348(\mathrm{NH}), 1603(\mathrm{C}=\mathrm{N})$, 1140 (C-O). ${ }^{1} \mathrm{H}$ NMR; 2.50-2.58 (m, 1H, C 4 pyrazole), 3.33-3.43 $\left(\mathrm{m}, 1 \mathrm{H}, \mathrm{C}_{4}\right.$ pyrazole), $3.84\left(\mathrm{~s}, 6 \mathrm{H}, 2 \mathrm{OCH}_{3}\right), 4.46-4.54(\mathrm{~m}, 1 \mathrm{H}$, $\mathrm{C}_{5}$ pyrazole), 6.69-7.78 (m, 12H, ArH), $9.18(\mathrm{~s}, 1 \mathrm{H}, \mathrm{N}-\mathrm{H}) . \mathrm{MS}$ $\mathrm{m} / \mathrm{z}(\%) ; 442.10\left(\mathrm{M}^{+}, 2.34\right), 440.10$ (1.12), 151.26 (100), 137.13 (4.45), 109.19 (25.58), 83.09 (22.24), 67.06 (21.13). Anal. Calcd for $\mathrm{C}_{24} \mathrm{H}_{22} \mathrm{~N}_{6} \mathrm{O}_{3}(\%)$ : C, 65.15; H, 5.01; $\mathrm{N}, 18.99$. Found: $\mathrm{C}, 65.43$; $\mathrm{H}, 5.34 ; \mathrm{N}, 19.07$.

5-(4-(5-(3,4,5-Trimethoxyphenyl)-1-phenyl-4,5-dihydro-1Hpyrazol-3-yl)phenoxy)-1H-tetrazole (12c)

Yield 63\%; mp $194-196{ }^{\circ} \mathrm{C} ;{ }^{1} \mathrm{H}$ NMR; $2.52-2.61$ (m, $1 \mathrm{H}, \mathrm{C}_{4}$ pyrazole), 3.32-3.40 (m, $1 \mathrm{H}, \mathrm{C}_{4}$ pyrazole $), 3.80(\mathrm{~s}, 3 \mathrm{H}$, $\left.\mathrm{OCH}_{3}\right), 3.87\left(\mathrm{~s}, 6 \mathrm{H}, \mathrm{OCH}_{3}\right), 4.72-4.79\left(\mathrm{~m}, 1 \mathrm{H}, \mathrm{C}_{5}\right.$ pyrazole $)$, 6.69-7.77 (m, 11H, ArH), $9.16(\mathrm{~s}, 1 \mathrm{H}, \mathrm{N}-\mathrm{H}) . \mathrm{MS} \mathrm{m} / \mathrm{z}(\%)$; $472.51\left(\mathrm{M}^{+}, 2.12\right), 470.51$ (1.12), 97.11 (25.58), 69.00 (49.48), 55.03 (100), 43.11 (55.04), 41.11 (36.14). Anal. Calcd. for $\mathrm{C}_{25} \mathrm{H}_{24} \mathrm{~N}_{6} \mathrm{O}_{4}(\%): \mathrm{C}, 63.55 ; \mathrm{H}, 5.12 ; \mathrm{N}, 17.79$. Found: $\mathrm{C}, 63.03$; $\mathrm{H}, 5.34 ; \mathrm{N}, 18.00$.

5-(4-(5-(4-Chlorophenyl)-4,5-dihydro-1H-pyrazol-3-yl)phenoxy)1-phenyl-1H-tetrazole $(\mathbf{1 2 d})$

Yield $62 \%$; mp $174-176^{\circ} \mathrm{C}$; IR; $3332(\mathrm{NH}), 1602$ $(\mathrm{C}=\mathrm{N}), 1172(\mathrm{C}-\mathrm{O}) .{ }^{1} \mathrm{H}$ NMR; $2.53-2.63\left(\mathrm{~m}, 1 \mathrm{H}, \mathrm{C}_{4}\right.$ pyrazole $)$, 3.31-3.42 ( $\mathrm{m}, \mathrm{H}, \mathrm{C}_{4}$ pyrazole $), 4.62-4.70\left(\mathrm{~m}, 1 \mathrm{H}, \mathrm{C}_{5}\right.$ pyrazole), 6.71-7.79 (m, 13H, ArH), 9.14 (s, 1H, N-H). ${ }^{13} \mathrm{C}$ NMR; 158.43, $154.62,151.86,150.53,141.70,131.80,130.84,129.38$, 129.31 (2C), 128.59 (2C), 128.43, 128.33, 127.22 (2C), 123.45 (2C), 121.98 (2C), 53.54 (H- $\underline{\mathrm{C}}-\mathrm{NH}), 40.63\left(\underline{\mathrm{CH}}_{2}\right) . \mathrm{MS} \mathrm{m} / \mathrm{z}$ (\%); $419.00\left(\mathrm{M}^{+}+2,1.39\right), 416.95\left(\mathrm{M}^{+}, 3.98\right), 258.07$ (89.51), 223.06 (45.59), 121.07 (100), 64.99 (55.62). Anal. Calcd for $\mathrm{C}_{22} \mathrm{H}_{17} \mathrm{ClN}_{6} \mathrm{O}(\%)$ : C, 63.39; H, 4.11; N, 20.16. Found: C, $63.43 ; \mathrm{H}, 4.34 ; \mathrm{N}, 20.07$.

5-(4-(5-(2-Bromophenyl)-4,5-dihydro-1H-pyrazol-3-yl)phenoxy)1-phenyl-1H-tetrazole (12e)

Yield 71\%; mp 185-186 ${ }^{\circ}$; IR; $3325(\mathrm{NH}), 1601$ $(\mathrm{C}=\mathrm{N}), 1171(\mathrm{C}-\mathrm{O}) .{ }^{1} \mathrm{H}$ NMR; 2.51-2.59 (m, 1H, $\mathrm{C}_{4}$ pyrazole), 3.34-3.45 (m, 1H, $\mathrm{C}_{4}$ pyrazole), 4.71-4.78 (m, 1H, $\mathrm{C}_{5}$ pyrazole), 6.69-7.78 (m, 13H, ArH), 9.17 (s, 1H, N-H). ${ }^{13} \mathrm{C}$ NMR; 158.45, $154.63,151.83,150.58,140.17,132.60,130.61,130.47,129.64$, 129.54 (2C), 129.29 (2C), 128.41, 127.59, 124.42 (2C), 121.95 (2C), 119.96, 53.43 (H- $\underline{\mathrm{C}}-\mathrm{NH}), 40.52\left(\underline{\mathrm{CH}}_{2}\right) . \mathrm{MS} \mathrm{m} / \mathrm{z}(\%) ; 463.05$ $\left(\mathrm{M}^{+}+2,2.78\right), 462.05\left(\mathrm{M}^{+}+1,1.67\right), 461.05\left(\mathrm{M}^{+}, 2.56\right), 264.19$ (83.96), 167.13 (100), 148.20 (50.04), 123.19 (87.84). Anal. Calcd for $\mathrm{C}_{22} \mathrm{H}_{17} \mathrm{BrN}_{6} \mathrm{O}(\%)$ : C, 57.28; H, 3.71; N, 18.22. Found: C, 57.43; H, 3.34; N, 18.07.

5-(4-(5-(4-Bromophenyl)-4,5-dihydro-1H-pyrazol-3-yl)phenoxy)1-phenyl-1H-tetrazole (12f)

Yield 60\%; mp 148-150 ${ }^{\circ}$; IR; $3328(\mathrm{NH}), 1604(\mathrm{C}=\mathrm{N})$, 1170 (C-O). ${ }^{1} \mathrm{H}$ NMR; 2.49-2.53 (m, 1H, C 4 pyrazole), 3.32-3.41 (m, 1H, $\mathrm{C}_{4}$ pyrazole), 4.77-4.80 (m, 1H, $\mathrm{C}_{5}$ pyrazole), 6.69-8.09 (m, 13H, ArH), 9.14 (s, 1H, N-H). ${ }^{13} \mathrm{C}$ NMR; 158.43 (Ar"-C-N), 154.43 (Ar'- $\underline{\mathrm{C}}-\mathrm{O}), 151.83(\mathrm{O}-\underline{\mathrm{C}}-\mathrm{N}), 150.56\left(\mathrm{Ar}^{\prime}-\underline{\mathrm{C}}=\mathrm{N}\right), 140.19$, $132.08,131.68$ (2C), 129.44, 129.38 (2C), 128.50 (2C), 127.51 (2C), 124.54 (2C), 121.95 (2C), 118.82, 53.43 (H-C-NH), 40.52 $\left(\underline{\mathrm{CH}}_{2}\right)$. Anal. Calcd for $\mathrm{C}_{22} \mathrm{H}_{17} \mathrm{BrN}_{6} \mathrm{O}(\%): \mathrm{C}, 57.28 ; \mathrm{H}, 3.71 ; \mathrm{N}$, 18.22. Found: C, 57.06; H, 3.90; N, 18.35. 
5-(4-(5-(4-Fluorophenyl)-4,5-dihydro-1H-pyrazol-3-yl)phenoxy)1-phenyl-1H-tetrazole (12g)

Yield $65 \%$; mp $170-171^{\circ} \mathrm{C}$; IR; $3333(\mathrm{NH}), 1603$ $(\mathrm{C}=\mathrm{N}), 1154(\mathrm{C}-\mathrm{O}) .{ }^{1} \mathrm{H}$ NMR; 2.48-2.55 (m, 1H, $\mathrm{C}_{4}$ pyrazole), 3.30-3.42 ( $\mathrm{m}, 1 \mathrm{H}, \mathrm{C}_{4}$ pyrazole), 4.84-4.98 (m, 1H, $\mathrm{C}_{5}$ pyrazole), 6.66-8.21 (m, 13H, ArH), 9.08 (s, 1H, N-H). ${ }^{13} \mathrm{C}$ NMR; 160.88, $158.39,154.32,151.87,150.53,139.55,130.46,129.31,129.28$ (2C), 127.62 (2C), 127.07 (2C), 124.67 (2C), 121.99 (2C), 115.63, 115.43, 53.21, $41.48\left(\mathrm{CH}_{2}\right)$. Anal. Calcd for $\mathrm{C}_{22} \mathrm{H}_{17} \mathrm{FN}_{6} \mathrm{O}(\%)$ : C, 65.99; H, 4.28; N, 20.99. Found: C, 66.06; H, 3.90; N, 21.35.

\section{5-(4-(5-(2-Nitrophenyl)-4,5-dihydro-1H-pyrazol-3-yl)phenoxy)- 1-phenyl-1H-tetrazole (12h)}

Yield 64\%; mp 170-172 ${ }^{\circ} \mathrm{C}$; IR; $3269(\mathrm{NH}), 1623(\mathrm{C}=\mathrm{N})$, 1174 (C-O). ${ }^{1} \mathrm{H}$ NMR; 2.50-2.57 (m, 1H, $\mathrm{C}_{4}$ pyrazole), 3.34-3.42 (m, 1H, $\mathrm{C}_{4}$ pyrazole), 4.51-4.59 (m, 1H, $\mathrm{C}_{5}$ pyrazole), 6.69-8.38 (m, 13H, ArH), 9.11 (s, 1H, N-H). ${ }^{13} \mathrm{C}$ NMR; 159.57, 156.55, $151.83,148.05,140.18,137.28,130.16,130.05,129.30,129.27$ (2C), 128.18 (2C), 127.01 (2C), 126.25, 123.48 (2C), 121.95 (2C), 53.54, 40.62. MS m/z (\%); $427.12\left(\mathrm{M}^{+}, 2.22\right), 111.13$ (26.69), 97.11 (37.25), 69.06 (45.04), 55.03 (100), 43.05 (43.37). Anal. Calcd for $\mathrm{C}_{22} \mathrm{H}_{17} \mathrm{BrN}_{6} \mathrm{O}(\%): \mathrm{C}, 61.82 ; \mathrm{H}, 4.01 ; \mathrm{N}, 22.94$. Found: C, 62.06; H, 3.90; N, 23.30.

N,N-Dimethyl-4-(3-(4-((1-phenyl-1H-tetrazol-5-yl)oxy) phenyl)4,5-dihydro-1H-pyrazol-5-yl)aniline (12i)

Yield 59\%; mp 185-186 ${ }^{\circ} \mathrm{C} ;{ }^{1} \mathrm{H}$ NMR; $2.54-2.62(\mathrm{~m}, 1 \mathrm{H}$, $\mathrm{C}_{4}$ pyrazole), $3.05\left(\mathrm{~s}, 6 \mathrm{H}, \mathrm{CH}_{3}\right), 3.36-3.43\left(\mathrm{~m}, 1 \mathrm{H}, \mathrm{C}_{4}\right.$ pyrazole), 5.00-5.15 (m, 1H, C 5 pyrazole), 6.69-7.77 (m, 13H, ArH), 9.07 (s, $1 \mathrm{H}, \mathrm{N}-\mathrm{H})$. Anal. Calcd. for $\mathrm{C}_{24} \mathrm{H}_{23} \mathrm{~N}_{7} \mathrm{O}(\%)$ : C, 67.75; H, 5.45; N, 23.04. Found: C, 67.99; H, 5.13; N, 23.30.

\section{Biological evaluation}

Cytotoxicity screening was evaluated by MTT assay, as previously reported (Ibrahim et al., 2014; Alarif et al., 2013). The used cells include human colon cancer (HCT-116), prostate cancer $(P C-3)$, breast cancer $(M C F-7)$ and normal kidney of African green monkey (Vero $B$ ) cell lines. The cell lines were obtained from American Type Culture Collection (ATCC). The cells were cultivated at $37^{\circ} \mathrm{C}$ and $10 \% \mathrm{CO}_{2}$ in a medium supplemented with $10 \%$ fetal bovine serum, 100 $\mathrm{IU} / \mathrm{ml}$ penicillin and $100 \mu \mathrm{g} / \mathrm{ml}$ streptomycin. Cisplatin (cisdiamineplatinum (II) dichloride) and 5-FU (5-fluorouracil) were obtained from Sigma and dissolved in $0.9 \%$ saline then stored as $8 \mathrm{mM}$ stock solution at $-20^{\circ} \mathrm{C}$. Then the synthesized compounds were solubilized in DMSO and stored at $-20^{\circ} \mathrm{C}$. The cells were seeded in 96-well plate as $5 \times 10^{4}$ cells $/ \mathrm{mL}(100$ $\mu \mathrm{L} /$ well). Serial dilutions of the tested compounds, Cisplatin and 5 -FU $(100,30,10,3.3,1.1$ or $0.36 \mathrm{ug} / \mathrm{ml})$ were added after overnight incubation of the cells at $37^{\circ} \mathrm{C}$ and $5 \% \mathrm{CO}_{2}$. DMSO was added to each well, and the solubilized formazan product was spectrophotometrically quantified with the help of a microplate reader, PowerWave XS (BioTek, Winooski, VT, USA), at $540 \mathrm{~nm}$. Each experiment was repeated three times and $\mathrm{IC}_{50}$ was calculated as the concentration that causes $50 \%$ inhibition of cell growth using GraphPad Prism (version 5.0, La Jolla, CA). The results were compared to the untreated cells
(DMSO without the tested compounds), and to cisplatin and 5-Fluorouracil (5-FU) as positive controls (Table 1). In order to find out whether the tested compounds are toxic to normal cells, their anti-proliferative activity against Vero- $B$ normal cell line was evaluated. Selectivity index was calculated by dividing the $\mathrm{IC}_{50}$ against cancer cell line over the $\mathrm{IC}_{50}$ against the normal cell line (Table 2).

$$
\mathrm{SI}=\left(\mathrm{IC}_{50}\right) \text { normal } /\left(\mathrm{IC}_{50}\right) \text { cancer. }
$$

\section{Molecular docking methodology}

Docking process was done on MOE software (MOE 2013.08 of Chemical Computing Group ULC). The newly prepared hybrids were built and optimized geometrically using ChemBioOffice, and a database file for the structures to be docked was prepared. Protein files were downloaded from the protein data bank (website: http://www.rcsb.org) and prepared for the docking process by keeping the binding sites flexible and adding water of solvation. PharmMapper server (Liu et al., 2010) was found helpful to determine the most suitable PDB files, where histone deacetylase 2 enzyme (HDAC2), cytochrome P450 17A1 and 5,10-methenyltetrahydrofolate synthetase (MTHFS) were chosen. Docking of twelve compounds to prove their binding mode with the selected proteins was done using 100 runs for each compound and Triangle marcher technique for placement stage, affinity $\mathrm{dG}$ for rescoring and forcefield method for refining. Docking scores were represented in Tables 3-5, and binding interactions of some representative compounds are shown in Figures 2-5.

\section{RESULTS AND DISCUSSION}

\section{Chemistry}

The reaction sequence used for the synthesis of the desired compounds is shown in Schemes 1, 2 and 3.

\section{Synthesis of compounds $\mathbf{5 a - c}$ and $\mathbf{6 a - c}$ (Scheme 1)}

The reported chalcone derivatives (3a-c) were prepared via Claisen-Schmidt condensation of substituted acetophenone derivatives $\mathbf{1 a}$ or $\mathbf{1 b}$ with the appropriate hydroxyl benzaldehyde (2a-c) in the presence of ethanolic $\mathrm{NaOH}(2.5 \%)$. The targeted tetrazole-containing hybrids (5a-c) were obtained via the wellknown Williamson ether synthesis by reacting 5-chloro-1phenyl-1H-tetrazole (4) with the prepared hydroxyl chalcone derivatives (3a-c) at room temperature for 24 hours while stirring in DMF and in the presence of anhydrous $\mathrm{K}_{2} \mathrm{CO}_{3}$. The IR spectra of these products (5a-c) showed carbonyl stretching vibrations of the enone fragments at $1665-1593 \mathrm{~cm}^{-1}$ and absorption bands at $1182-1155 \mathrm{~cm}^{-1}$ for ether link (C-O). ${ }^{1} \mathrm{H}$ NMR spectrum of compound $\mathbf{5 b}$ demonstrated characteristic singlet peak assigned to 6 protons of two methoxy groups at $\delta 3.80 \mathrm{ppm}$. The vinylic protons resonated as two doublets within the aromatic region with $J$ value $15 \mathrm{~Hz}$. This observation indicates the trans configuration of the enone chalcone structure (Silverstein et al., 1991). The structure of compound $\mathbf{5 b}$ was supported by ${ }^{13} \mathrm{C}$ NMR spectroscopy that showed a signal at $188.32 \mathrm{ppm}$ for the carbonyl carbon, while the two methoxy carbons appeared as two signals at 56.16 and 56.13 . 
Table 1: In vitro antiproliferative activities of the designed compounds.

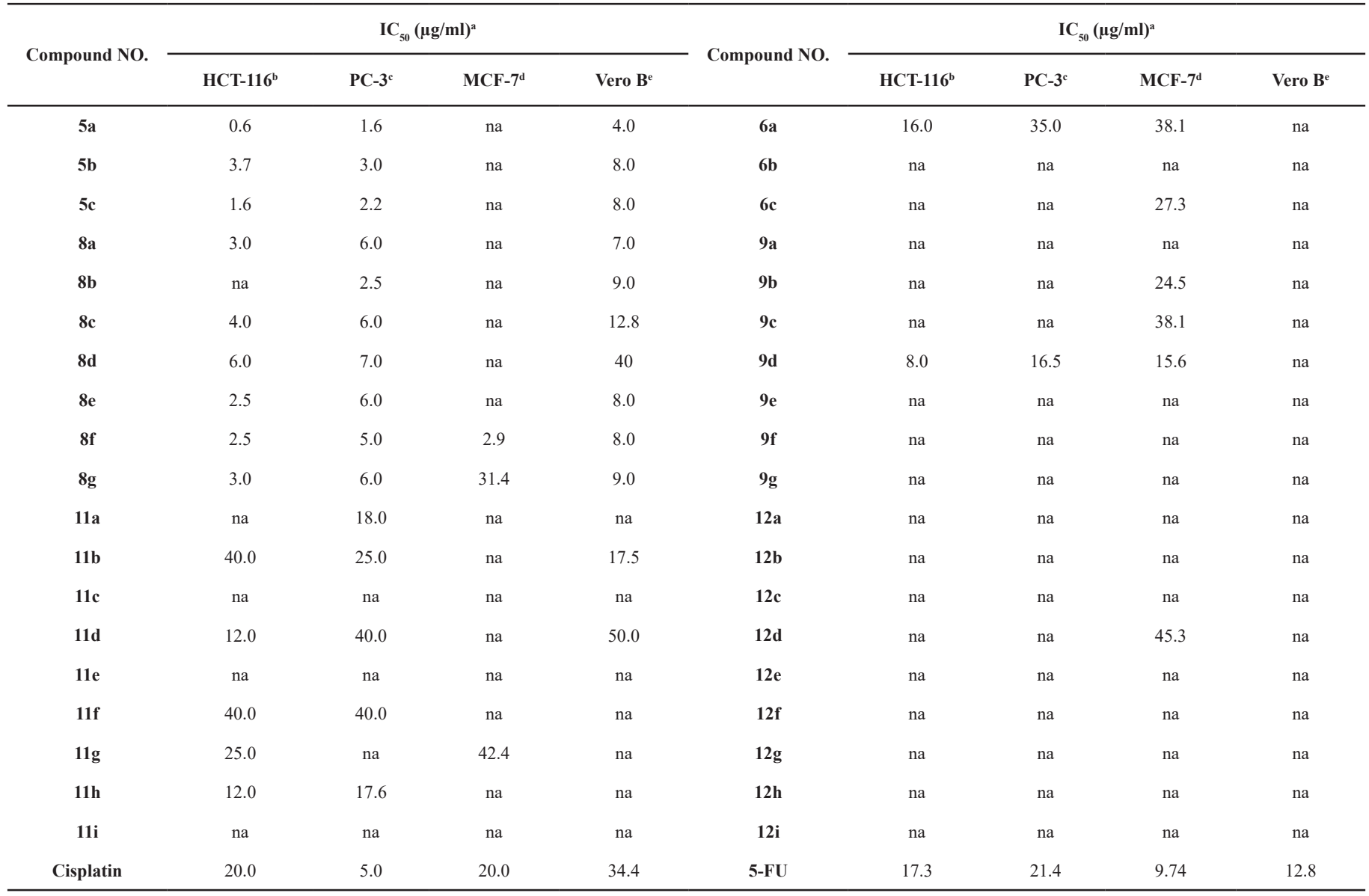

${ }^{a} \mathrm{IC}_{50}$ : Compound concentration required to inhibit cell proliferation by $50 \%$.

${ }^{\mathrm{b}}$ Colon cancer cell line; ${ }^{\mathrm{c}}$ Prostate cancer cell line; ${ }^{\mathrm{d}}$ Breast cancer cell line; ${ }^{\mathrm{e}}$ Normal African green monkey kidney cell line. na: not active till $50 \mu \mathrm{g} / \mathrm{ml}$.

Table 2: Selectivity indices of the active compounds.

\begin{tabular}{|c|c|c|c|c|c|c|c|}
\hline \multirow{2}{*}{ Compound NO. } & \multicolumn{3}{|c|}{ Selectivity index (SI) ${ }^{\mathrm{a}}$} & \multirow{2}{*}{ Compound NO. } & \multicolumn{3}{|c|}{ Selectivity index (SI) ${ }^{\mathrm{a}}$} \\
\hline & HCT-116 ${ }^{\mathrm{b}}$ & PC-3 $3^{\mathrm{c}}$ & MCF- $7^{d}$ & & HCT-116 & PC-3 $3^{\mathrm{c}}$ & $\mathrm{MCF}-7^{\mathrm{d}}$ \\
\hline $5 \mathbf{b}$ & 2.16 & 2.66 & - & $9 b$ & - & - & $>2.04$ \\
\hline $5 c$ & 5.00 & 3.63 & - & $9 \mathrm{c}$ & - & - & $>1.31$ \\
\hline $8 \mathbf{a}$ & 2.33 & 1.16 & - & $11 \mathrm{~b}$ & 0.43 & 0.70 & - \\
\hline $8 \mathbf{b}$ & - & 3.60 & - & $11 d$ & 4.16 & 1.25 & - \\
\hline $8 c$ & 3.20 & 2.13 & - & $11 \mathrm{f}$ & $>1.25$ & $>1.25$ & - \\
\hline $8 d$ & 6.66 & 5.71 & - & $11 \mathrm{~g}$ & $>2.00$ & - & $>1.17$ \\
\hline
\end{tabular}

a Selectivity index: $\mathrm{SI}=\left(\mathrm{IC}_{50}\right)$ normal/ $\left(\mathrm{IC}_{50}\right)$ cancer.

${ }^{\mathrm{b}}$ Colon cancer cell line; ${ }^{\mathrm{c}}$ Prostate cancer cell line; ${ }^{\mathrm{d}}$ Breast cancer cell line. 
Table 3: Binding scores $(\mathrm{Kcal} / \mathrm{mol})$ of the twelve docked compounds with HDAC2 in comparison to reference inhibitor $\mathrm{N}$-(2-aminophenyl)benzamide.

\begin{tabular}{cccc}
\hline Compound No. & Binding scores & Compound No. & Binding scores \\
\hline $\mathbf{5 a}$ & -8.54584217 & $\mathbf{6 a}$ & -6.35466719 \\
$\mathbf{5 b}$ & -11.0169601 & $\mathbf{6 b}$ & -10.7547264 \\
$\mathbf{8 d}$ & -11.2120924 & $\mathbf{9 d}$ & -10.7752962 \\
$\mathbf{8 e}$ & -9.7349453 & $\mathbf{9 e}$ & -9.6193018 \\
$\mathbf{8 g}$ & -9.52019119 & $\mathbf{9 g}$ & -8.99661255 \\
$\mathbf{1 1 a}$ & -11.9166155 & Reference ligand. & -10.7447565 \\
$\mathbf{1 1 b}$ & -8.08154964 & & \\
\hline
\end{tabular}

Table 4: Binding scores $(\mathrm{Kcal} / \mathrm{mol})$ of the twelve docked compounds with $\mathrm{CY}$ $\mathrm{P} 17 \mathrm{~A} 1$ in comparison to reference drug TOK-001.

\begin{tabular}{cccc}
\hline Compound No. & Binding scores & Compound No. & Binding scores \\
\hline $\mathbf{5 a}$ & -7.758 & $\mathbf{6 a}$ & -7.483 \\
$\mathbf{5 b}$ & -10.088 & $\mathbf{6 b}$ & -8.740 \\
$\mathbf{8 d}$ & -9.4827 & $\mathbf{9 d}$ & -9.414 \\
$\mathbf{8 e}$ & -8.312 & $\mathbf{9 e}$ & -9.356 \\
$\mathbf{8 g}$ & -9.072 & $\mathbf{9 g}$ & -8.870 \\
$\mathbf{1 1 a}$ & -8.418 & Reference ligand. & -8.518 \\
$\mathbf{1 1 b}$ & -8.472 & & \\
\hline
\end{tabular}

Table 5: Binding scores $(\mathrm{Kcal} / \mathrm{mol})$ for the twelve docked compounds with MTHFS in comparison to reference 10-formyltetrahydrofolate.

\begin{tabular}{cccc}
\hline Compound No. & Binding scores & Compound No. & Binding scores \\
\hline $\mathbf{5 a}$ & -9.943 & $\mathbf{6 a}$ & -9.395 \\
$\mathbf{5 b}$ & -9.173 & $\mathbf{6 b}$ & -10.523 \\
$\mathbf{8 d}$ & -10.218 & $\mathbf{9 d}$ & -9.726 \\
$\mathbf{8 e}$ & -10.294 & $\mathbf{9 e}$ & -12.534 \\
$\mathbf{8 g}$ & -10.646 & $\mathbf{9 g}$ & -10.819 \\
$\mathbf{1 1 a}$ & -8.911 & Reference ligand. & -11.102 \\
$\mathbf{1 1 b}$ & -9.161 & & \\
\hline
\end{tabular}

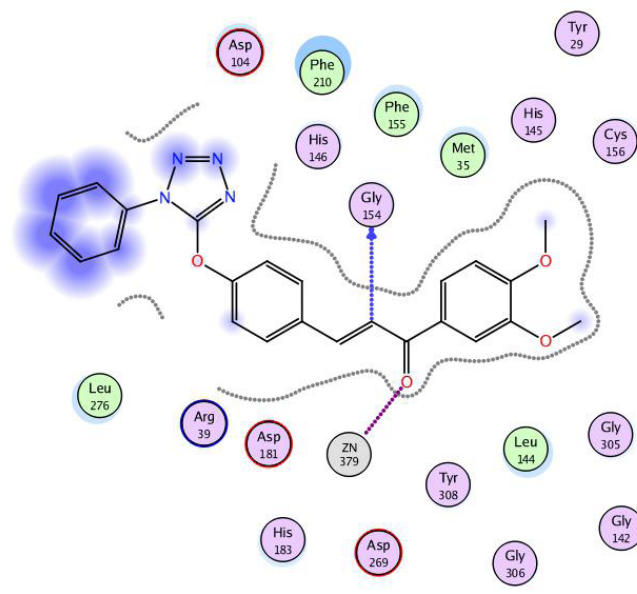

Fig. 2: Binding of HDAC2 with compound (5b).

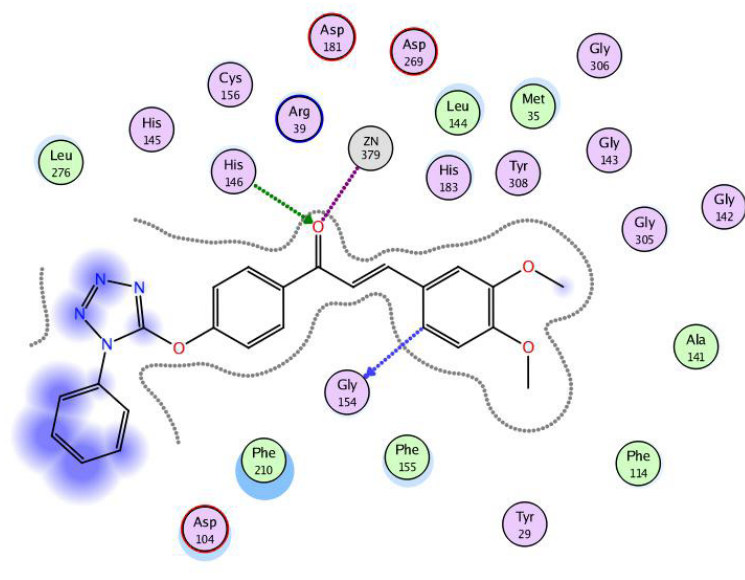

Fig. 3: Binding of HDAC2 with compound (11b).

Compounds 6a-c were prepared by reacting $\alpha, \beta$ unsaturated ketones (5a-c) with an excess of hydrazine hydrate $(95 \%)$ in ethanol, at reflux temperature. The successful synthesis of pyrazoline derivatives was verified by the micro-analytical and spectral data. IR spectra of 6a-c showed the disappearance of the carbonyl stretching vibration of compounds 5a-c and the appearance of absorption bands at 3347-3336 cm $\mathrm{cm}^{-1}$ and $1604-1590$ $\mathrm{cm}^{-1}$ associated with $\mathrm{NH}$ and $\mathrm{C}=\mathrm{N}$ groups of pyrazoline ring. The ${ }^{1} \mathrm{H}$ NMR spectrum of compound $\mathbf{6 c}$, as a representative example, showed the chiral proton at $\mathrm{C}_{5}$ of the pyrazoline ring as a doublet of doublet at 4.87 with a coupling constant 11.7 and $5.1 \mathrm{~Hz}$ due to vicinal coupling with the methylene protons $\left(\mathrm{CH}_{2}\right)$ at $\mathrm{C}_{4}$ of the pyrazoline ring. The $\mathrm{CH}_{2}$ protons appeared as two distinct doublets of doublet at $\delta 2.79$ and $3.12 \mathrm{ppm}$. Each proton has a germinal and vicinal coupling with coupling constant of 18.1 and $11.7 \mathrm{~Hz}$ for one proton and 18.1 and $5.1 \mathrm{~Hz}$ for the other. In addition, a $\mathrm{D}_{2} \mathrm{O}$ exchangeable $\mathrm{NH}$ proton singlet was observed at $\delta 9.18$. Mass spectrum of the pyrazoline derivatives displayed molecular ion peaks inconsistent with the assigned molecular weight. For compound $\mathbf{6 b}$, a molecular ion peak was observed at $\mathrm{m} / \mathrm{z}, 442.45$ in agreement with its formula $\mathrm{C}_{24} \mathrm{H}_{22} \mathrm{~N}_{6} \mathrm{O}_{3}$.

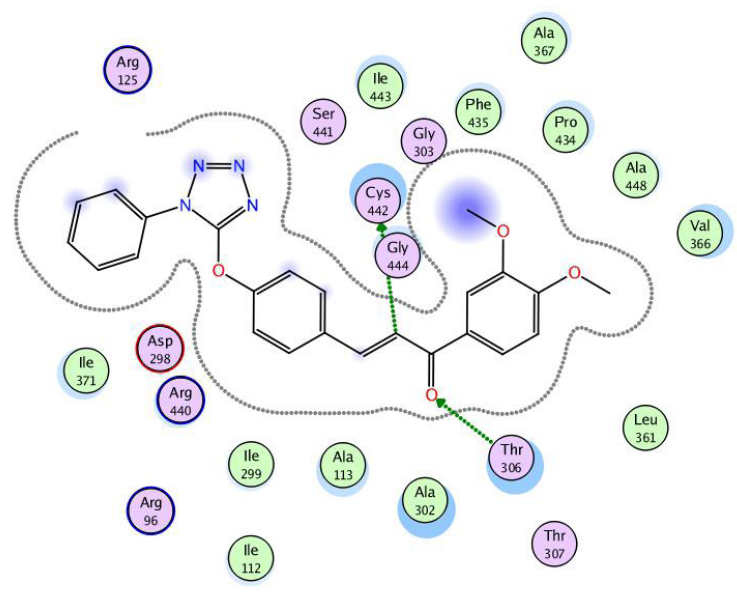

Fig. 4: Ligand interactions in the binding site of CYP17A1 with compound (5b). 


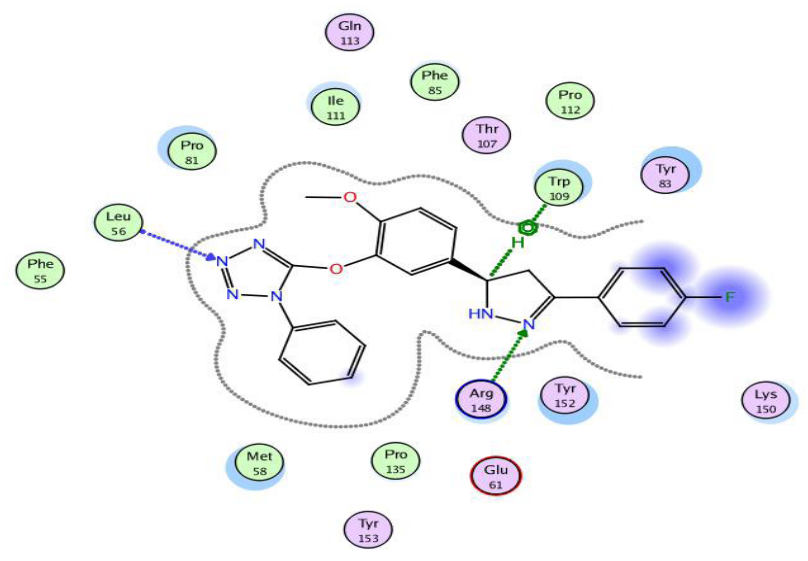

Fig. 5: Ligand interactions in the binding site of MTHFS with compound (9e).

\section{Synthesis of compounds $8 \mathbf{a - g}$ and $\mathbf{9 a - g}$ (Scheme 2)}

The reported compound 3-methoxy-4-((1-phenyl$1 H$-tetrazol-5-yl)oxy) benzaldehyde (7) was obtained via $O$-alkylation of 4-hydroxy-3-methoxybenzaldehyde (vanillin) (2b) with 5-chloro-1-phenyl-1H-tetrazole (4) in DMF in the presence of anhydrous $\mathrm{K}_{2} \mathrm{CO}_{3}$. Compound 7 was then reacted with 1 equivalent of substituted acetophenones, 2-acetylthiophene or 2-acetylfuran (1c-i) in an ethanolic solution of $\mathrm{NaOH}(2.5 \%)$ followed by acidification with $\mathrm{HCl}$ to afford $\alpha, \beta$-unsaturated carbonyl compounds (8a-g). Nucleophilic cycloaddition reaction of compounds (8a-g) with hydrazine monohydrate $(95 \%)$ in ethanol gave the corresponding pyrazolines (9a-g). The IR spectra of the later compounds showed bands at $3350-3347 \mathrm{~cm}^{-1}$ of the $(\mathrm{N}-\mathrm{H})$ group and $1604-1574 \mathrm{~cm}^{-1}$ of $(\mathrm{C}=\mathrm{N})$ group. ${ }^{1} \mathrm{H}$ NMR spectrum of compound $9 \mathrm{~b}$ revealed the presence of a broad singlet at $\delta 9.23 \mathrm{ppm}$ of the N-H proton; and three multiplet bands at $\delta$ 2.35-2.41, 3.31-3.40 and 4.55-4.61 ppm integrated for the two $\mathrm{C}_{4}$ protons at and the $\mathrm{C}_{5}$ proton of the pyrazoline ring, respectively. A molecular ion peak was detected at $\mathrm{m} / \mathrm{z} 442.16$, in accordance with the molecular formula of compound $9 \mathbf{a}$.

\section{Synthesis of compounds $11 \boldsymbol{a}-\boldsymbol{i}$ and $\mathbf{1 2 a} \mathbf{- i}$ (Scheme 3)}

The starting compound 1-(4-((1-phenyl-1H-tetrazol5-yl)oxy)phenyl) ethan-1-one (10) was prepared as previously reported, by reacting 5-chloro-1-phenyl- $1 H$-tetrazole (4) and 4-hydroxyacetone (1j) in DMF in the presence of anhydrous $\mathrm{K}_{2} \mathrm{CO}_{3}$ as a catalyst. Claisen-Schmidt condensation of differently substituted benzaldehydes (2d-i) with compound $\mathbf{1 0}$ was carried out in ethanolic $\mathrm{NaOH}(2.5 \%)$, followed by acidification with $\mathrm{HCl}$ afforded the target compounds 1-(4-((1-phenyl-1H-tetrazol-5-yl) oxy)phenyl)-3-(un)substituted phenylprop-2-en-1-ones (11a-i). In ${ }^{1} \mathrm{H}$ NMR spectrum of compound 11c, the protons of three methoxy groups resonated as two singlets at $\delta 3.79\left(\mathrm{OCH}_{3}\right)$ and 3.88 $\left(2 \mathrm{OCH}_{3}\right)$. These methoxy carbons appeared as three signals in ${ }^{13} \mathrm{C}$ NMR spectrum at 56.13, 56.03 and $55.71 \mathrm{ppm}$. Also, the structure of compound 11i was confirmed by its ${ }^{1} \mathrm{H}$ NMR spectroscopy that showed a singlet peak assigned to two methyl groups at $\delta$ 3.43 ppm representing the dimethylamino moiety. The pyrazoline compounds 12a-i were prepared by reacting $\alpha, \beta$-unsaturated ketones (11a-i) with hydrazine hydrate (95\%) in refluxing ethanol.
Again, microanalytical and spectral data of these pyrazolines were inconsistent with the proposed structures. A molecular ion peak at $\mathrm{m} / \mathrm{z} 472.51$ represented the molecular weight of compound 12c was observed in its mass spectrum.

\section{BIOLOGICAL ACTIVITY}

\section{In vitro antiproliferative screening}

Several approaches have been utilized to measure the viability of cells and determine cell growth via staining with different vital dyes. In the present work, the 3-(4,5-dimethylthiazol2-yl)-2,5-diphenyltetrazolium bromide (MTT assay) was used to assess the antiproliferative activity of the newly synthesized tetrazole-chalcone/pyrazoline hybrids. This assay depends on the ability of viable cells to reduce the yellow tetrazolium salt (MTT) to a purple product. Three mammalian cell lines, namely: the colon cancer $(H C T-116)$, prostate cancer $(P C-3)$ and breast cancer $(M C F-7)$ cell lines, and the African green monkey kidney (Vero- $B)$ normal cell line were used. $\mathrm{The}_{\mathrm{IC}}$ of the tested compounds along with that of the reference drugs Cisplatin and 5-Fluorouracil are shown in Table 1 and selectivity indices of the active compounds are shown in Table 2.

Regarding the antiproliferative activity against colon HCT-116 cell line, eleven chalcone derivatives showed higher activity, with $\mathrm{IC}_{50}$ ranging from 0.6 to $12 \mu \mathrm{g} / \mathrm{ml}$, than that of cisplatin $\left(\mathrm{IC}_{50}=20 \mu \mathrm{g} / \mathrm{ml}\right)$ and $5-\mathrm{FU}\left(\mathrm{IC}_{50}=17.3 \mu \mathrm{g} / \mathrm{ml}\right)$. Compounds 5a and $\mathbf{5 c}$ were identified as the most potent compounds at $\mathrm{IC}_{50}=0.6$ and $1.6 \mu \mathrm{g} / \mathrm{ml}$ with selectivity index $=6.66$ and 5.00 , respectively. Compounds 8e and $\mathbf{8 f}$ showed their effect against colon HCT-116 cell line at $\mathrm{IC}_{50}=2.5 \mu \mathrm{g} / \mathrm{ml}$ that represents eight and seven times the activity of the reference drugs cisplatin and 5-FU, respectively, while compounds $8 \mathbf{a}$ and $\mathbf{8 g}\left(\mathrm{IC}_{50}=3 \mu \mathrm{g} / \mathrm{ml}\right)$ were proved to be six and five times these reference drugs. Other derivatives that showed better $\mathrm{IC}_{50}$ values than both cisplatin and 5-FU included $\mathbf{5 b}, \mathbf{8 c}$, 8d, 11d, and $\mathbf{1 1 h}\left(\mathrm{IC}_{50}\right.$ of 3.6-12 $\left.\mu \mathrm{g} / \mathrm{ml}\right)$. On the other hand, only two pyrazoline derivatives, 6a and 9d, displayed equal or better activity against colon $H C T-116$ cell lines than the reference drugs, with $\mathrm{IC}_{50}$ values of 16 and $8 \mu \mathrm{g} / \mathrm{ml}$. In addition, both compounds 6a and 9d have no cytotoxic activities against the normal Vero $B$ cell line till a concentration of $50 \mu \mathrm{M}$.

Regarding the activity against prostate $P C-3$ cell line, compounds 5a-c and $\mathbf{8 b}$ exhibited $\mathrm{IC}_{50}$ values of 1.6-3 $\mu \mathrm{g} / \mathrm{ml}$, better than that of the reference drugs cisplatin $\left(\mathrm{IC}_{50}=5 \mu \mathrm{g} / \mathrm{ml}\right)$ and 5-FU $\left(\mathrm{IC}_{50}=21.4 \mu \mathrm{g} / \mathrm{ml}\right)$ with selectivity indices ranging from 2.50 to 3.63 . In addition, compounds, 8a and $\mathbf{8 c - g}$ showed nearly comparable activities, as revealed from their $\mathrm{IC}_{50}(5-7 \mu \mathrm{g} / \mathrm{ml})$, to cisplatin $\left(\mathrm{IC}_{50}=5\right.$ $\mu \mathrm{g} / \mathrm{ml})$, but higher than 5-FU ( $\left.\mathrm{IC}_{50}=21.4 \mu \mathrm{g} / \mathrm{ml}\right)$. On the other hand, none of the pyrazoline derivatives displayed comparable activity with cisplatin; but only 9d displayed a better effect than that of 5-FU with no effect on the normal Vero B cell line.

Among the tested compounds, only the chalcone derivative $\mathbf{8 f}$ showed remarkable antiproliferative activity against breast $M C F-7$ cell line with $\mathrm{IC}_{50}=2.9 \mu \mathrm{g} / \mathrm{ml}$ and $\mathrm{SI}=2.75$; while the pyrazoline 9d displayed an $\mathrm{IC}_{50}=15.6 \mu \mathrm{g} / \mathrm{ml}$ and showed selectivity against breast $M C F-7$ cancer cell line versus the normal Vero B cell line.

The above mentioned biological results indicated the presence of antiproliferative activity in the chalcone compounds, 
rather than their corresponding pyrazoline derivatives and most of the active compounds exerted high selectivity indices. Eight of the tested chalcone compounds (5a-c, 8a, 8c, 8e-g) were proved to be of better or equal effect than the reference drugs against two tested cell lines, while only two pyrazoline derivatives (6a and 9d), showed only weak or modest activity against the three tested cancer cell lines. The activity emerged mostly with chalconetetrazole hybrids, where the chalcone enone system appears essential, and tetrazole moiety is connected at the $\beta$-carbon of the enone system.

In such a group of active compounds 5a-c and 8a-g, the substitution pattern at phenyl A of chalcone system did not show great prevalence, except that the presence of a $\mathrm{CH}_{3}$ group, with both hydrophobic and electron-donating properties, at para position, negatively affected the activity against colon HCT-116 cancer cell lines.

Compound 5a, in which the 1-phenyl tetrazolyloxy moiety is incorporated at ortho position of the phenyl ring linked to the $\beta$-carbon of the enone system, exerted superior activity relative to the other tested chalcone derivatives, against the tested cell lines; while compound $\mathbf{5} \mathbf{c}$ is the second most potent compound, where three methoxy groups are present at the two chalcone phenyl rings.

Interchanging the enone system in compound $\mathbf{5 b}$ to give 11b resulted in a negative impact on the activity. The same pattern was observed with all other reversed chalcone derivatives 11a and $11 \mathrm{c}-\mathbf{i}$.

The growth inhibition activity against breast $M C F-7$ cell lines appeared with compound $\mathbf{8 f}$ indicating the beneficial effect of the 2-thienyl groups on the action against this type of cancer cell lines. Compound $\mathbf{8 f}$ has a broad spectrum of activity against the three tested cancer cell lines.

\section{DOCKING STUDIES}

Molecular docking studies were carried out for twelve compounds (biologically active and inactive), to help to elucidate which portions of the molecules are critical for antiproliferative activity.

\section{Docking with histone deacetylase 2 enzyme (HDAC2) (PDB: 3MAX)}

Compounds were tested for binding with HDAC2 that is important in colon cancer cells (Bressi et al., 2010). The HDAC2 active site consists of the catalytic machinery, a lipophilic 'tube' which leads from the surface to this machinery, and a 'foot pocket' immediately adjacent to the machinery. The overall binding scores of the tested compounds with HDAC2 in comparison to reference inhibitor N-(2-aminophenyl)benzamide are shown in Table 3. Compounds $\mathbf{5 b}$ and $\mathbf{8 d}$ displayed higher binding scores than the reference ligand. Figure 2 shows the importance of showing the importance of the $\alpha-\beta$ unsaturated carbonyl moiety, in compound $\mathbf{5 b}$, in binding to $\mathrm{Zn}$ atom and amino acid Gly154 of the binding site of HDAC2.

However, compound 11a, which is a reversed chalcone structure of compounds $\mathbf{5}$ and $\mathbf{8}$, exhibited higher binding score than other derivatives, though it was proved inactive in the in vitro biological testing. Figure 3 represents the binding of compound 11b to the active site of HDAC2, where no interaction between the electron rich double bond and Gly154 was observed, but instead a hydrophobic interaction with the adjacent phenyl group, and binding of the carbonyl group with $\mathrm{Zn}$ atom and His146. In the biological evaluation, compound 11b displayed weak activity against colon HCT-116 cell lines.

\section{Docking with Cytochrome P450 17A1 (CYP 17A1) (PDB: 3SWZ)}

Cytochrome P450 17A1 catalyzes the biosynthesis of androgens in humans (DeVore and Scott, 2012); and prostate cancer cells proliferate in response to androgen steroids. The overall binding scores of the twelve compounds with CYP17A1 in comparison to reference drug TOK-001are displayed in Table 4 , where the highest binding score of -10.088 was observed with compound $\mathbf{5 b}$. Interaction of chalcone tested compound $\mathbf{5 b}$ in the binding site of CYP17A1 is shown in Figure 4, where binding of the $\alpha-\beta$ unsaturated carbonyl moiety was observed with Cys 442 and Thr306 residues. This pattern of interaction goes consistently with the observed in vitro biological activity.

\section{Docking with 5,10-Methenyltetrahydrofolate synthetase (MTHFS) (PDB: 3HY3)}

MTHFS regulates the flow of carbon through the onecarbon metabolic network, which supplies essential components for the growth and proliferation of cells. Inhibition of MTHFS in human MCF-7 breast cancer cells has been shown to arrest the growth of cells (Wu et al., 2009). The overall binding scores for the tested compounds with MTHFS in comparison to reference 10-formyltetrahydrofolate are shown in Table 5, where the pyrazoline compound 9e displayed the highest negative binding score (-12.534) among the tested compounds. Ligand interaction in the binding site of MTHFS with this pyrazoline (9e) is shown in Figure 5, where the tetrazole and pyrazole rings interact with Leu56, Trp109, and Arg148. Yet, this compound (9e) was proved inactive against $M C F-7$ cell lines in the in vitro antiproliferative evaluation.

\section{CONCLUSION}

Nineteen chalcone-tetrazole hybrids and their cyclic pyrazoline-tetrazole structures were prepared and biologically evaluated. Eight of the tested chalcone compounds (5a-c, 8a, 8c, 8e-g) showed significant antiproliferative activities, with $\mathrm{IC}_{50}$ values better than, or comparable to, that of the reference drugs, cisplatin, and 5-FU, against HCT-116 and PC-3 cell lines with high selectivity towards cancer cell lines.

The activity appeared mostly with chalcone-tetrazole hybrids, where the chalcone enone system appears essential; and tetrazole moiety is connected at the $\beta$-carbon of the enone system. Interchanging the enone system in compounds $\mathbf{5}$ and $\mathbf{8}$ to give $\mathbf{1 1}$ or its cyclization into a pyrazoline structure (6 and 9) has, generally, a negative impact on the activity.

The substitution pattern at phenyl A of chalcone system, in the active compounds $\mathbf{5}$ and $\mathbf{8}$ did not show great prevalence. However, the presence of a 2-thienyl group (8f) has a beneficial effect on the growth inhibition activity against breast $M C F-7$ cell lines. Compound $\mathbf{8 f}$ has a broad spectrum of activity against the three tested cancer cell lines.

Compound $\mathbf{5 b}$ binds to HDAC2 and CYP17A1 enzymes with a binding score of -11.017 and $-10.088 \mathrm{Kcal} / \mathrm{mol}$ 
respectively, inconsistent with its antiproliferative activity against colon $H C T-116$, and prostate $P C-3$ cell lines $\left(\mathrm{IC}_{50}=3.7\right.$ and 3.0). On the other hand, no interaction between the electron rich double bond of compound $\mathbf{1 1 b}$, a reversed chalcone structure, and Gly154 in the active site of HDAC2 was observed.

Although the pyrazoline compound 9e didn't show any activity in the present in vitro study, it had good binding scores with the amino acids residues in the MTHFS enzyme.

\section{REFERENCES}

Alarif WM, Abdel-Lateff A, Al-Abd AM, Basaif SA, Badria FA, Shams M, Ayyad S-EN. Selective cytotoxic effects on human breast carcinoma of new methoxylated flavonoids from Euryops arabicus grown in Saudi Arabia. Eur J Med Chem. 2013; 66:204-210.

Arshad M, Bhat AR, Pokharel S, Kim J, Lee EJ, Athar F, Choi I. Synthesis, characterization and anticancer screening of some novel piperonyl-tetrazole derivatives. Eur J Med Chem. 2014; 71:229-236.

Bayomi SM, El-Kashef HA, El-Ashmawy MB, Nasr MNA, ElSherbeny MA, Abdel-Aziz NI, El-Sayed MA-A, Suddek GM El-Messery SM, Ghaly MA Synthesis and Biological Evaluation of New Curcumin Analogues as Antioxidant and Antitumor Agents: Molecular Modeling Study. Eur J Med Chem. 2015; 101:584-694.

Bayomi SM, El-Kashef HA, El-Ashmawy MB, Nasr MNA, El-Sherbeny MA. Badria FA, Abou-zeid LA, Ghaly MA, Abdel-Aziz NI. Synthesis and biological evaluation of new curcumin derivatives as antioxidant and antitumor agents. Med. Chem. Res. 2013; 22(3):1147-1162.

Bayomi SM, Moustafa MA, Maarouf AR, Abutaleb MH. Design, Synthesis, Biological Activity and Molecular Modeling of New Heterocyclic Tetrazole Derivatives. J Am Sci. 2016; 12(1):40-56.

Bhaskar VH, Mohite PB. Synthesis, characterization and evaluation of anticancer activity of some tetrazole derivatives. J. Optoelectronics and Biomedical Materials. 2010; 2(4):249-259.

Bressi JC, Jennings AJ, Skene R, Wu Y, Melkus R, Jong RD, O'Connell S, Grimshaw CE, Navre M, Gangloff AR. Exploration of the HDAC2 foot pocket: Synthesis and SAR of substituted N-(2-aminophenyl) benzamides. Bioorg Med Chem Lett. 2010; 20(10):3142-3145.

Buonerba F, Lepri S, Goracci L, Schindler BD, Seo SM, Kaatz GW, Cruciani G. Improved Potency of Indole Based NorA Efflux Pump Inhibitors: From Serendipity toward Rational Design and Development. J Med Chem. 2017; 60:517-523.

Cai CY, Rao L, Rao Y, Guo J-X, Xiao Z-Z, Cao J-Y, Huang Z-S, Wang B. Analogues of xanthones-Chalcones and bis-chalcones as $\alpha$-glucosidase inhibitors and anti-diabetes candidates. Eur J Med Chem. 2017; 130:51-59.

de Vasconcelos A, Campos VF, Nedel F, Seixas FK, Dellagostin OA, Smith KR, de Pereira CMP, Stefanello F M, Collares T, Barschak AG. Cytotoxic and apoptotic effects of chalcone derivatives of 2-acetyl thiophene on human colon adenocarcinoma cells. Cell Biochem Funct. 2013; 31(4):289-297.

DeVore NM, Scott EE. Structures of cytochrome P450 17A1 with prostate cancer drugs abiraterone and TOK-001. Nature. 2012; 482(7383):116-119.

Eisa HM, Moustafa MA, El-Kerdawy MM. Synthesis of some novel tetrazole derivatives as potential antimicrobial agents. Pak J Sci Ind Res. 1990; 33(10):417-420.

Fischer JM, Robbins SB, Al-Zoughool M, Kannamkumarath SS, Stringer SL, Larson JS, Caruso JA, Talaska G, Stambrook PJ, Stringer JR. Co-mutagenic activity of arsenic and benzo[a]pyrene in mouse skin; Mutat Res. 2005; 588(1):35-46.

Ibrahim A, Sobeh M, Ismail A, Alaa A, Sheashaa H, Sobh M, Badria F. Free-B-Ring flavonoids as potential lead compounds for colon cancer therapy. Mol Clin Oncol. 2014; 2(4):581-585.

Jedhe GS, Paul D, Gonnade RG, Santra MK, Hamel E, Nguyen TL, Sanjayan GJ. Correlation of hydrogen-bonding propensity and anticancer profile of tetrazole-tethered combretastatin analogues. Bioorg Med Chem Lett. 2013; 23(16):4680-4684.

Liu X, Ouyang S, Yu B, Liu Y, Huang K, Gong J, Zheng S, Li Z, Li H, Jiang H. PharmMapper server: a web server for potential drug target identification using pharmacophore mapping approach. Nucleic Acids Res. 2010; 38(2):W609-W614.

Molecular Operating Environment (MOE), 2013.08; Chemical Computing Group ULC, 1010 Sherbooke St. West, Suite \#910, Montreal, QC, Canada, H3A 2R7; 2017

Murad MA, Abdel-Aziz M, Abuo-Rahma G, Farag HH. Design, synthesis and anticancer activity of nitric oxide donating/chalcone hybrids. Eur J Med Chem. 2012; 54:907-913.

Nitulescu GM, Draghici C, Olaru O. New Potential Antitumor Pyrazole Derivatives: Synthesis and Cytotoxic Evaluation. Int J Mol Sci. 2013; 14(11):21805.

Pan Y, Chen Y, Li Q, Yu X, Wang J, Zheng J. The Synthesis and Evaluation of Novel Hydroxyl Substituted Chalcone Analogs with in Vitro Anti-Free Radicals Pharmacological Activity and in Vivo Anti-Oxidation Activity in a Free Radical-Injury Alzheimer's Model. Molecules. 2013; 18(2):1693-1703.

Pathak V, Ahmad I, Kahlon AK, Hasanain M, Sharma S, Srivastava KK, Sarkar J, Shankar K, Sharma A, Gupta A. Syntheses of 2-methoxyestradiol and eugenol template based diarylpropenes as nonsteroidal anticancer agents. RSC Advances. 2014; 4(66):35171-35185.

Sankappa Rai U, Isloor AM, Shetty P, Pai KSR, Fun HK. Synthesis and in vitro biological evaluation of new pyrazole chalcones and heterocyclic diamides as potential anticancer agents. Arab. J. Chem. 2015; 8(3):317-321.

Sharma V, Chaudhary A, Arora S, Saxena AK, Ishar MPS. $\beta$-Ionone derived chalcones as potent antiproliferative agents. Eur J Med Chem. 2013; 69:310-315.

Silverstein RM, Bassler GC, Morrill TC. Spectroscopic Identification of Organic Compounds, John Wiley and sons (5th Ed.), Inc. NY. 1991.

Viale M, Anzaldi M, Aiello C, Fenoglio C, Albicini F, Emionite L, Gangemi R, Balbi A. Evaluation of the antiproliferative activity of three new pyrazole compounds in sensitive and resistant tumor cell lines. Pharmacol Rep. 2013; 65(3):717-723.

Vogelstein B, Papadopoulos N, Velculescu VE, Zhou S, Diaz LA, Kinzler KW. Cancer Genome Landscapes. Science. 2013; 339(6127):15461558. Website: http://www.rcsb.org.

Wu D, Li Y, Song G, Cheng C, Zhang R, Joachimiak A, Shaw N, Liu Z-J. Structural Basis for the Inhibition of Human 5,10-Methenyltetrahydrofolate Synthetase by N10-Substituted Folate Analogues. Cancer Res. 2009; 69(18):7294-7301.

Yuan JW, Wang S-F, Luo Z-L, Qiu H-Y, Wang P-F, Zhang X, Yang Y-A, Yin Y, Zhang F, Zhu H-L. Synthesis and biological evaluation of compounds which contain pyrazole, thiazole and naphthalene ring as antitumor agents. Bioorg Med Chem Lett. 2014; 24:2324-2328.

\section{How to cite this article:}

Abd ElMonaem HS, Abdel-Aziz NI, Morsy MA, Badria FA, ElSenduny F, El-Ashmawy MB, Moustafa MA. Synthesis, In Vitro Antiproliferative Evaluation and Molecular Docking of New tetrazole-chalcone and tetrazole-pyrazoline Hybrids. J App Pharm Sci, 2018; 8(05): 075-087. 